\title{
Gaze behavior in face comparison: The roles of sex, task, and symmetry
}

\author{
REGINE ARMANN AND ISABELLE BÜLTHOFF \\ Max Planck Institute for Biological Cybernetics, Tübingen, Germany
}

\begin{abstract}
Knowing where people look on a face provides an objective insight into the information entering the visual system and into cognitive processes involved in face perception. In the present study, we recorded eye movements of human participants while they compared two faces presented simultaneously. Observers' viewing behavior and performance was examined in two tasks of parametrically varying difficulty, using two types of face stimuli (sex morphs and identity morphs). The frequency, duration, and temporal sequence of fixations on previously defined areas of interest in the faces were analyzed. As was expected, viewing behavior and performance varied with difficulty. Interestingly, observers compared predominantly the inner halves of the face stimuli-a result inconsistent with the general left-hemiface bias reported for single faces. Furthermore, fixation patterns and performance differed between tasks, independently of stimulus type. Moreover, we found differences in male and female participants' viewing behaviors, but only when the sex of the face stimuli was task relevant.
\end{abstract}

Looking at a face is the most reliable way to identify an individual, but in addition to recognizing familiar faces, we can derive other important information from the face of a person we do not know. Faces are central in human interactions because they provide critical information about the age, sex, identity, mood, and intention of another person. Numerous psychophysical studies have investigated various aspects of the process of face perception (see, e.g., Bruce \& Young, 1986; Farah, Wilson, Drain, \& Tanaka, 1998; for a review, see Bruce \& Young, 1998). Recently, eyetracking studies have provided new insight into the way we encode and perceive faces (e.g., Pearson, Henderson, Schyns, \& Gosselin, 2003; Stacey, Walker, \& Underwood, 2005; Williams, Senior, David, Loughland, \& Gordon, 2001).

Since the eyes see only a small part of a visual scene at high resolution at a time, they are constantly moving to scan interesting features with the fovea. Records of eye movements (Loftus \& Mackworth, 1978; Yarbus, 1967) have shown that foveal vision is directed mainly to visual elements containing information essential to the observer during perception. Henderson, Williams, and Falk (2005) showed that eye movements play an important functional role in face learning, since restricting the eye gaze of observers to the center of a face during a learning phase clearly impairs later recognition performance.

Pearson et al. (2003) recorded observers' gazes in different face-perception experiments and found that not all information in the face images was looked at with the same frequency and duration, and that the task that observers were asked to perform especially influenced viewing behavior. When they were instructed to classify faces by their sex, observers looked longer at the eyes of the face images; when they were given a face-identification task, the fixation time was more distributed all over the face images. In the same study, the mouth was looked at more often and longer when the mood of the face was to be specified. This task dependency of eye movements when faces are being viewed has been corroborated by studies using the "bubble method" (see, e.g., Schyns, Bonnar, \& Gosselin, 2002), which have shown that, for example, eyes alone are "diagnostic" for sex decisions, but not for face identification. Both studies pointed out the importance of task-dependent attention allocation, as opposed to the stimulus-driven saliency involved in face perception.

Barton, Radcliffe, Cherkasova, Edelman, and Intriligator (2006) have suggested that internal representations resulting from familiarity with a face influence scanning patterns. Their study showed eye-movement sequences for famous faces to be more idiosyncratic - that is, more different from each other - than the more standardized scanning patterns for unknown faces.

Furthermore, the two halves of a face have been found to be of unequal importance: Observers tend to base their responses predominantly on the information on the left in sex classification (see, e.g., Butler et al., 2005) as well as in face memory tasks (Gilbert \& Bakan, 1973; Mertens, Siegmund, \& Grüsser, 1993), or on the judgment of facial expressions (e.g., Christman \& Hackworth, 1993). In general, this perceptual bias toward the left hemiface is interpreted as a consequence of a right-hemisphere specialization for face processing (see, e.g., Barton et al., 2006; Kanwisher, McDermott, \& Chun, 1997).

R. Armann, regine.armann@tuebingen.mpg.de 
All of these findings demonstrate that eye movements can tell us something about how faces are perceived and represented. Although observers are not always consciously aware of it, they allocate their attention (i.e., eye gaze) to specific features of a face (and not to others), or in a specific pattern. Note that most of the eyetracking studies on face perception that have been cited so far investigated gaze behavior of observers during learning or recognition of single-face images. In that case, the scanning pattern might depend on memory representations of known face identities or of general facial characteristics (e.g., expressions) that are compared with the face image, depending on the paradigm (task). Hence, only one side of the comparison - that is, the presented stimulus - is available to the experimenter and can be manipulated, whereas internal representations remain inaccessible. Yet, in everyday situations, one also happens to compare faces of people physically present or to match the face of a person to a photograph. When the visual world is interacted with in such a way, the stimuli being compared remain visually available to the observer; thus, in an experiment using a direct comparison task, both sides of the comparison can be manipulated and are accessible for analysis. Furthermore, in the aforementioned studies that are most relevant to our work, observers were highly familiar (Pearson et al., 2003; Schyns et al., 2002) with the faces they had to identify or classify by their sex, or at least they saw the same faces over and over again (Gosselin \& Schyns, 2001). Although familiarity with the test face is needed in an identification task, it is not required in order to classify a face's sex; in that case, familiarization was nevertheless done in these studies to ensure that the participants had the same levels of experience with all faces. The results of these studies might thus pertain more to the specifics of gaze behavior when known faces are inspected than to information about diagnostic features generally used when observers identify or classify faces. On this account, we investigated eye movements when observers looked at two unfamiliar faces presented side by side; in this setting, there is no necessary reference to some internal memory representation. We assume that eye fixations to specific facial features indicate the importance given to these features for the task at hand, whereas specific scanning patterns reflect different strategies of information retrieval (e.g., on the basis of local features, on the basis of relations between features, or on the basis of whole faces).

Thus, the first question that motivated the present study was whether-despite the differences between single-face experiments and face-pair comparisons that have been mentioned, and when strictly unfamiliar test faces were used-our participants would consider the same key facial features diagnostic as was the case for those reported in earlier studies. If this were the case, our results would not only confirm but extend the validity of these earlier findings to a general level.

Second, we asked how observers compared the two faces of a pair: They could compare each facial feature with the corresponding feature on the other face, analogous to the object-by-object strategy that has been found in comparative visual search tasks (Galpin \& Underwood, 2005), or they could compare the faces as a "whole." The latter would be indicated, for example, by more extensive scanning patterns on each face before comparison with the other face, and/or by relatively few comparison saccades between faces. On the basis of the repeatedly shown lefthemiface bias in face-perception tasks, we would also assume that observers compare the left half-faces of the two stimuli with each other, rather than the right half-faces.

Third, since there is some evidence in the literature indicating that differences in the performance of men and women occur in some face-perception tasks, we balanced the number of male and female participants in our experiments. A female advantage for recognizing faces (Guillem \& Mograss, 2005; Lewin \& Herlitz, 2002; Rehnman \& Herlitz, 2007) and classifing them by sex (O'Toole, Peterson, \& Deffenbacher, 1996) has been shown, as have differences in adaptation to the sex of faces depending on the observers' own sex (Webster, Kaping, Mizokami, $\&$ Duhamel, 2004). We therefore compared the data of these two subgroups of participants in order to correlate potential performance differences with different looking behaviors in male and female observers.

Since we could control both sides of the comparison, we manipulated the difficulty of the tasks systematically by varying the similarity of the two faces shown in a trial. That way, we could investigate whether changes in difficulty lead to changes in viewing strategy (and if so, whether they lead to similar changes in different tasks). Previous findings have shown that, for example, the common difficulty in recognizing other-race faces correlates with a more feature-based processing of those stimuli, as compared with more configural processing of own-race faces - that is, a task in which we naturally have more expertise (see, e.g., Michel, Caldara, \& Rossion, 2006; Michel, Rossion, Han, Chung, \& Caldara, 2006).

The facial manipulations we investigated were related to identity and sex, since these are natural, socially meaningful, and well-studied properties of human faces. Observers performed two same-different tasks with face pairs that were manipulated in either sex or identity, respectively.

A third task was a two-alternative forced choice task (2AFC) asking participants to point to the more feminine face of each pair. The reason for adding this task was that we wanted to know whether facial information is accessed differently when task instructions change but the stimuli, as well as the facial characteristic of interest, remain the same. For that purpose, we exploited an apparent inconsistency in the face literature about the perception of sex: On the one hand, there is the very fast and robust ability to categorize faces by their sex (see, e.g., Bruce \& Young, 1986; Bruyer, Galvez, \& Prairial, 1993; Deffenbacher, Hendrickson, O'Toole, Huff, \& Abdi, 1998); on the other hand, it has been shown that observers are very poor at noticing and remembering variations of sex-related facial information (Bülthoff \& Newell, 2004). We hypothesized that the difficulty of the latter task may result from its unnaturalness: People generally do not change their sex, even ever so slightly, on a regular basis. From this hypothesis, 
we further assumed that these differences in performance might be associated with different viewing behavior of observers when classifying faces by sex or when looking for sex-related variations. To investigate our assumption, we contrasted the same-different task on face pairs manipulated by sex - a task that is indeed not very natural — with the more intuitive task of asking the participant to point to the more feminine face of a pair. Since we were not interested in differences between judgments of maleness or femaleness but in differences between male and female participants doing the same task, we did not include a task in which participants were asked to find the more masculine face of a pair.

Many of the studies about face perception that have been mentioned so far used small numbers of face images that were derived from normal photographs equated in size and contrast. In our present study, control over the face stimuli was improved by using an in-house face database containing over 300 three-dimensional computerreconstructed laser scans of real male and female heads. Using this facilitated the generation of a large number of stimuli with homogeneous, well-controlled properties (e.g., orientation, size, lighting conditions).

By using the "flexible face model" of Blanz and Vetter (1999), we could selectively modify specific face properties (such as sex and identity) of our face stimuli. This model represents faces as location vectors in a multidimensional face space (see Valentine, 1991). Thus, the manipulation of the position of a face along just one dimension in terms of this face space results in varying only this property, whereas the other characteristics of the face remain unchanged. Another advantage of this flexible model over standard morph-based codes is that it includes correspondences for all sample points (i.e., approximately 70,000 vertices) rather than just a subset of key feature points. This enables us to capture subtle shape and texture variations in the internal regions of the faces and to generate natural-looking stimuli.

In summary, the viewing behavior and discrimination performance of male and female observers comparing two faces were assessed. The similarity of the two faces of a pair was varied parametrically within each task, and we assumed that task difficulty would vary accordingly; the faces being compared differed in sex or identity characteristics, and in the case in which sex was manipulated, two kinds of comparisons were performed. We wanted to test whether the same facial information that is considered diagnostic in single-face studies would also be found when the stimuli to be compared were unfamiliar and remained visible - thus, when the information that was essential for solving the task could be accessed as needed and when it was unnecessary to encode or access a detailed internal memory representation. Furthermore, we investigated how observers would compare the two faces of a pair, looking for differential scanning patterns depending on the testing conditions. We also explored whether different viewing patterns might emerge when the same facial characteristic (sex) was dealt with under different task instructions by building on a seeming contradiction in the literature about sex perception. Finally, in view of the sex differences reported in face-recognition literature, we investigated whether male and female participants would examine face pairs in a different way.

\section{METHOD}

\section{Stimuli}

Static face images were derived from three-dimensional laser scans collected in the face database of the Max Planck Institute for Biological Cybernetics (http://faces.kyb.tuebingen.mpg.de). The full-frontal two-dimensional face images derived from these heads were between 460 and 550 pixels in height and between 320 and 390 pixels in width, which corresponds roughly to the natural size of a face from chin to hair line (about $20 \times 14 \mathrm{~cm}$ ). At a viewing distance of $56 \mathrm{~cm}$ (a distance at which adults typically interact; Baxter, 1970), a face covered a visual area of about $20^{\circ} \times 14^{\circ}$ of visual angle. All faces were shown in color (24-bit color depth).

External features such as ears and hair were removed, and the faces were free from other cues, such as makeup or facial hair. To eliminate irregular edges resulting from the removal of the scalp, which could be used as a cue to distinguish the faces, we gradually faded the upper-face contour into the background color for each face by use of a standard alpha blending algorithm with a transparency mask that was empirically determined on the average head.

A morphing algorithm developed by Blanz and Vetter (Blanz, 2000; Blanz \& Vetter, 1999) was used to manipulate the faces according to two parameters - that is, either their identity or their sex, separately. For "sex morphing," faces were manipulated along the "sex vector" calculated between the average of all male and the average of all female faces of the scanned population of heads. Nine evenly distributed face morphs (steps of $10 \%$ change) were then created between each face identity and its opposite-sex version. Identity morphs were obtained by computing a linear combination of the vectors of two faces of the same sex, creating nine evenly distributed morphs between the two original faces. Since each participant performed two tasks with sex-manipulated faces and one task with identity-manipulated faces, more faces ( 95 female and 95 male) were used to create sex morphs than to create identity morphs (70 female and 70 male). No face identity was used twice in the whole experiment. Figure 1 shows two examples of "morph continua."

\section{Apparatus}

The stimuli were presented using E-Prime (Version 1.1, Psychology Software Tools, Inc.) on a desktop computer linked to a 17-in. CRT monitor (resolution, $1,024 \times 768$ pixels; refresh rate, $75 \mathrm{~Hz}$ ). Eye movements were recorded with an iView X SMI dark pupil remote eyetracker system. Retinal and corneal reflections induced by an infrared source were recorded at a frequency of $60 \mathrm{~Hz}$ to obtain participants' points of fixation on the computer screen (error of resolution: $0.5^{\circ}-1^{\circ}$ ). Participants were sitting in front of the monitor displaying the test stimuli at a distance of $56 \mathrm{~cm}$ and placed their head on a chinrest with a head band to avoid movements. A button box (Psychology Software Tools, Inc.) was used for collecting responses

\section{Design}

In all trials, two face images were presented side by side on the monitor. At the designated distance, it was not possible to focus on both faces simultaneously. Because previous work had demonstrated that visual information retrieving is in general limited to a small region of about $4^{\circ}$ around the fixation point (Henderson, Williams, Castelhano, \& Falk, 2003), we were sure to prevent participants from perceiving all of the information on the screen "at a glance." However, the visual angle covered by both faces (about $28^{\circ} \times 20^{\circ}$ ) was small enough to allow participants to keep their heads still when fixating on any part of them. Each face pair consisted of two face 

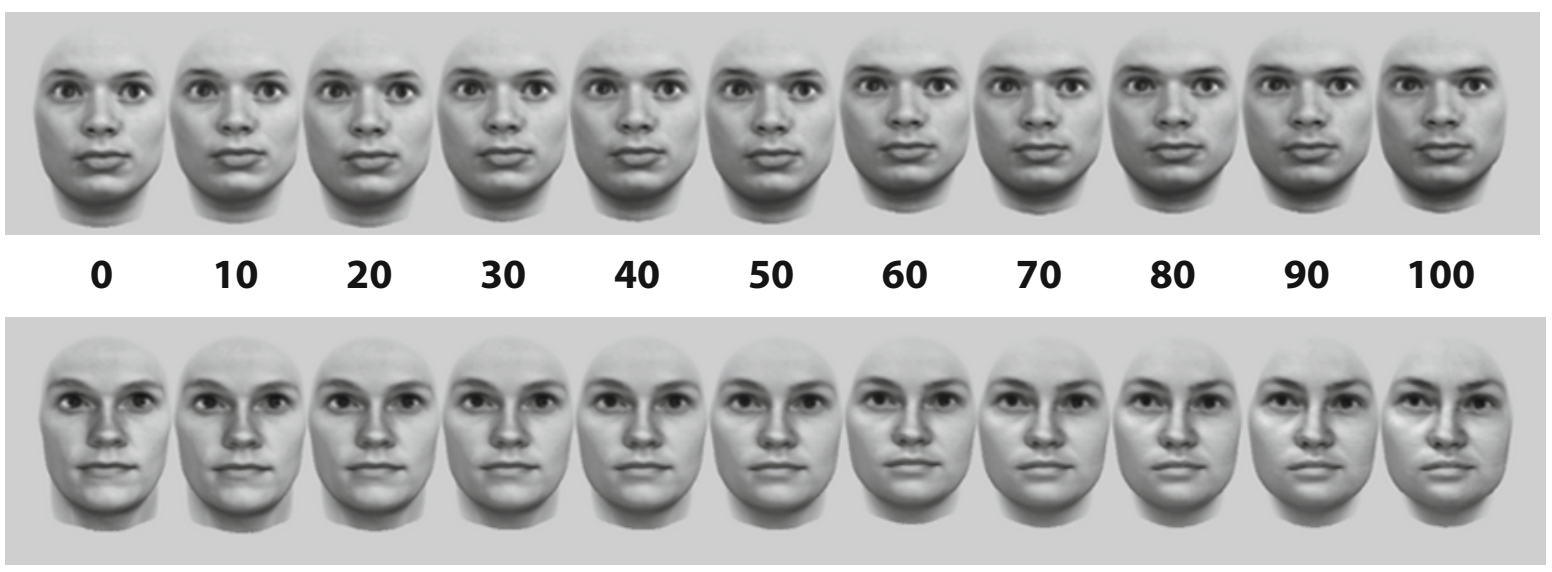

Figure 1. Examples of sex (upper row) and identity (lower row) morph continua. The original face ("0") is on the left; numbers indicate the percentage of morphing toward the opposite sex or identity (see text for more details). The face stimuli were presented in color.

images from the same morph continuum (see Figure 1, showing a sex continuum consisting of one face in its original appearance and its associated sex morphs). The faces of a pair could be identical or different. The smaller the distance between the "morph levels" of the two faces in a pair, the more similar the faces were to each other in that trial.

Participants were asked to perform three different experimental tasks. For the "sex task" and the "identity task" (the same-different tasks), face stimuli from either the sex or the identity morph continua were used, respectively. In the "feminine task" (a 2AFC task asking participants to point to the more feminine face of each pair), the same kind of sex morphs as in the sex task was used, but only different face pairs were shown. Participants performed the sex task and the identity task (order counterbalanced across participants). Since the feminine task was different, it was always performed last: We did not want to affect participants' intuitive strategies in the same-different tasks as a result of their experience with the $2 \mathrm{AFC}$ task. To uncover any effect of practice in the feminine task, we conducted a control experiment (see below).

Each of the tasks was based on a within-subjects repeated measures (RM) design with "morph distance" between the faces of a pair as the independent variable; the dependent variables were response time (RT), accuracy (ACC), number of fixations, duration of fixation, time spent within a region of interest, and a range of other eyeposition measures that will be specified in the Results section.

To rule out face familiarity effects within the tasks, since they might affect not only identification and fixation patterns (see the introduction) but also sex discrimination if both are linked (as was suggested, e.g., by Bülthoff \& Newell, 2004; Ganel \& GoshenGottstein, 2002; and Rossion, 2002), each face morph continuum (original face plus morphs; see Figure 1) was used only once in the whole experiment.

In a study using face stimuli derived from the same database and manipulated with the same morphing algorithm, Bülthoff and Newell (2004) found that people were not able to distinguish between an original face and a sex morph of that face at a morph distance level of $20 \%$. Therefore, in order to provide about the same difficulty in the two same-different tasks, and because of the time constraints of the eyetracking technique, we showed only face pairs differing by a morph distance of $30 \%$ or greater in the sex task and the feminine task.

For both same-different tasks, in half of the trials, face stimuli were paired with themselves ("same pairs"); in the other half, the face morphs were paired with the original face of their morph series ("different pairs"). Two stimuli sets were created for the sex task by using each face once in a "same-pair" trial in one set and once in a "different-pair" trial in the other set. The same procedure was implemented for the faces used in the identity task. The sets were counterbalanced across participants. In the feminine task, all faces were paired with the original face of their morph continuum, since only "different pairs" were required. Two stimulus sets for this task were created by assigning each face to one morph level in one set and to another morph level in the second set. For the control experiment (Experiment 2), a third stimulus set for the feminine task was created with faces that had been used before in the sex and identity tasks (see below). The sex (identity) task consisted of 144 (140) same and different trials. In order to test participants for no more than $1 \mathrm{~h}$ (longer durations usually result in a drop of eyetracking accuracy due to fatigue, causing, e.g., excessive blinking by the participants), the feminine task was designed with 44 trials only. The total time of the experiment, including instruction, training, and calibration procedures (around $1 \mathrm{~h}$ ) was determined in several pilot experiments.

\section{Participants}

All participants were between 18 and 37 years old and had normal or corrected-to-normal vision. Most of them were paid volunteers who were recruited via the Max Planck Institute Subject Database; a few were members of the Max Planck Institute. All of them were naive as to the purpose of the experiments and participated only once.

\section{Procedure}

Participants were given oral instructions before entering the test cubicle. Instruction consistency was ensured by using a written checklist. A calibration procedure was carried out before the experiment and in between the tasks. After each task, participants left the room for a few minutes to make sure they relaxed. For the same-different tasks, participants were instructed to decide in each trial whether the two faces were identical or not, and they were told in which aspect they had to look for differences (identity or sex). In the feminine task, they were told to find the more feminine face of each face pair. No feedback was given during testing, but it was provided in a training phase preceding each task (see below). After the experiment, participants were asked several questions about their performance (e.g., "Did you find the tasks equally easy/difficult to solve?"; "What do you think is the difference between male and female faces?") to verify that they had performed the tasks correctly. Details of the main and the control experiments are given below.

Experiment 1. Thirty-two participants completed this experiment. Fifteen of them were female. Trials were organized as follows: A fixation cross (shown for $750 \mathrm{msec}$ ) preceded the test stimulus 
(two faces side by side). The face images disappeared as soon as a response button was pressed, and they were followed by a blank screen for $600 \mathrm{msec}$.

Before each experimental block, participants went through a training block with feedback. On the basis of the mean RTs measured in a pilot experiment (which differed from the main experiment only insofar as there was no time limit), decision time was restrained to $10 \mathrm{sec}$ in the sex task and to $7 \mathrm{sec}$ in the identity task, whereas participants had a maximum of $5 \mathrm{sec}$ in the feminine task. These time restraints were designed to avoid extreme outliers in RT but were defined broadly enough not to influence participants' response strategies. If no response was given before the end of that period, the face stimuli disappeared and the trial was excluded from the analysis. Participants were informed about the limited RT and could experience it in the training block. Later, in the Results section, we will present observers' gaze behavior (presented as percentage of fixations to the different areas of interest [AOIs]) from Experiment 1 and from the aforementioned pilot experiment together (Figure 6), to demonstrate that there was indeed no effect of time restriction on viewing behavior. Furthermore, the much shorter time to respond in the feminine task did not lead to a higher loss of trials due to time-outs in this task as compared with the other two. Calculated across all participants, $3.9 \%$ of trials per task were lost in the sex task $(S E M=0.7)$, in which observers had the longest time to respond, $3.6 \%$ in the identity task $(S E M=1.0)$, and $3.7 \%$ in the feminine task $(S E M=1.2)$, which had the strictest time limit.

Experiment 2. Seventeen participants took part in this experiment. Eight of them were female. Participants performed only the feminine task. Only psychophysical data were collected. The RT was limited to $5 \mathrm{sec}$ as in Experiment 1, and participants went through the same training procedure.

\section{Data Acquisition and Reduction}

E-Prime software recorded psychophysical and stimulus data for every trial. The trials in which participants did not answer within the restricted time frame were not taken into account for the analysis of psychophysical and eyetracking data. Eyetracking data were recorded as $x$-and $y$-pixel coordinates of point of regard on the screen during each trial. Recording started at the beginning of the trial and stopped with the participant's response. Trials with more than $15 \%$ missing data points (due to blinks or tracking instability) were excluded from the analysis, as were participants' data for a whole task if they had more than $15 \%$ missing trials. Two participants who reported that their response was based on cues not related to the required execution of the tasks were completely excluded from the analysis. Of the 30 remaining participants, 2 more were completely excluded for total loss of tracking data; 10 participants' data for all tasks and 18 participants' data for one or two of the tasks could be used. Altogether, we retained the data of 20 participants for the sex and the feminine tasks (10 feminine, 10 masculine), and 18 participants for the identity task ( 8 feminine, 10 masculine). Thus, neither was there a gender skew, nor were the data of one task disproportionately removed from the analysis.

We did not restrain our analyses to only those trials in which participants had answered correctly, for two reasons: (1) Trial number (i.e., statistical validity) would have been largely reduced, and, moreover, this reduction would have disproportionately affected the different tasks; and (2) we were not interested in the most effective strategy but in the strategy participants considered the most appropriate for a certain task.

\section{Data Analysis and Statistics}

Position values for key feature points in the face (e.g., medial and lateral corners of the eyes, tip of the nose, corners of the mouth) were defined on one reference face. Since the faces in our database were represented in correspondence to each other, we could use the morphable model algorithm (see above) to define automatically the same points on each face individually. On the basis of these points,

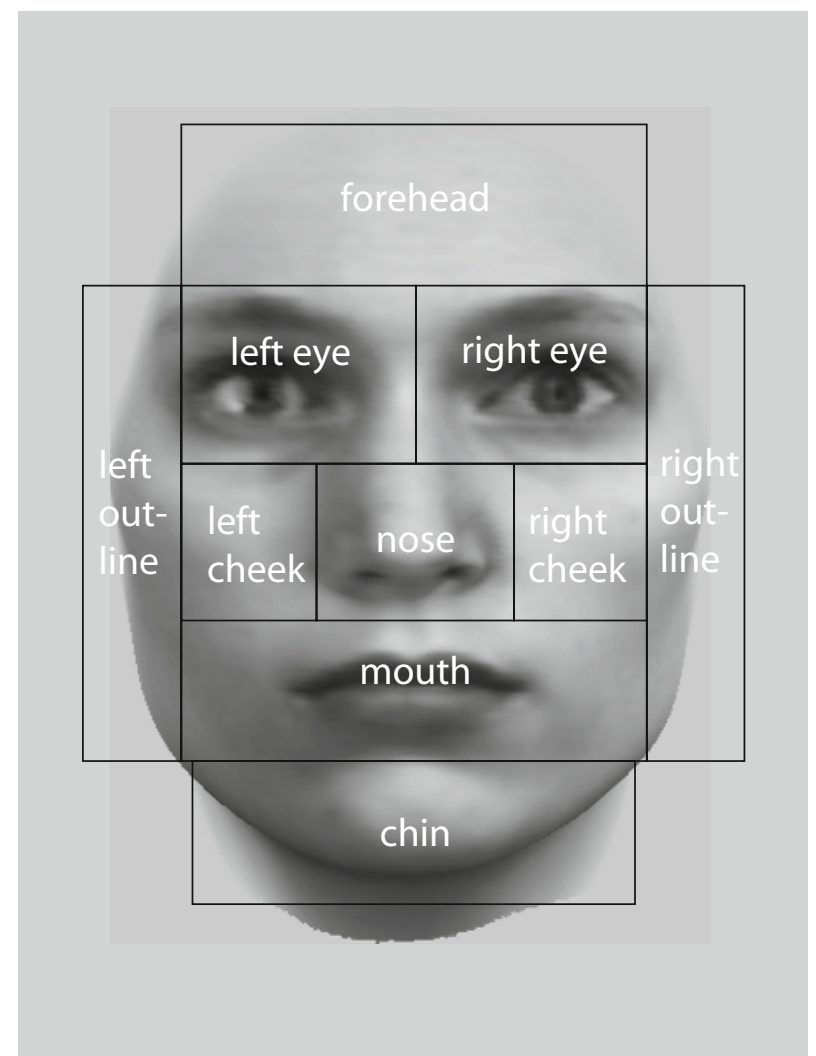

Figure 2. Ten areas of interest were defined on each face individually, using key feature points (e.g., corners of the eyes, tip of the nose, etc.).

all face images were individually segmented into 10 AOIs. See Figure 2 for a schematic example.

We used the freeware MATLAB toolbox "iLab" (Gitelman, 2002) for filtering blinks and calculating fixations from the iView X trial data. A fixation was defined as a set of consecutive gaze coordinates, confined within a diameter of $1^{\circ}$ of visual field for a duration of $100 \mathrm{msec}$ or more (Williams et al., 2001). The coordinate values of each fixation determined in which AOI the fixation was made.

All data were analyzed using MATLAB (Versions 6.5.1 and 7.1), Excel 2000, and SPSS (Version 12.0) for Windows. Once the position, time, and length of all fixations had been calculated, a set of analyses was conducted to investigate various aspects of viewing behavior. These analyses will be described along with the results in the next section.

The analyses within tasks were based on the GLM repeated measures analyses (fixed-effects) model in SPSS. Since the tasks differed in paradigms (same-different vs. 2AFC) and stimuli used (no "same pairs" in the feminine task; not all morph distance levels in the sex and feminine tasks), their data were primarily compared qualitatively but not quantitatively — that is, by performing statistical tests within tasks. The more assumption-free nonparametric Wilcoxon signed rank test was used to compare across tasks for some explicitly chosen normalized measures.

\section{RESULTS}

\section{Psychophysical Data}

Repeated measures ANOVAS were conducted on performance data (ACC) and on RTs for each task separately. 


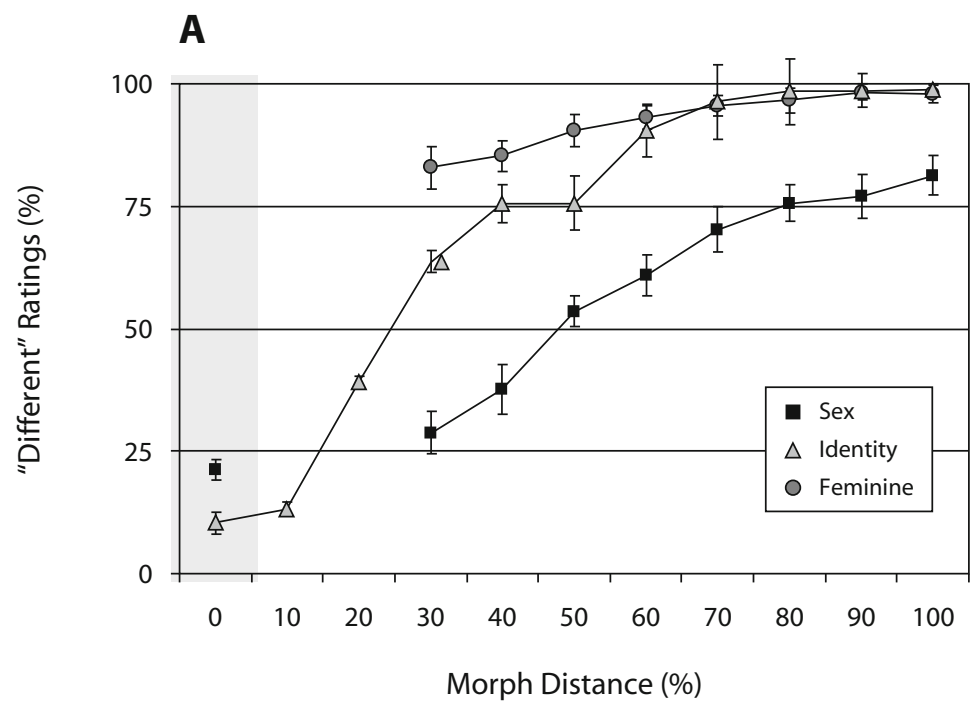

B

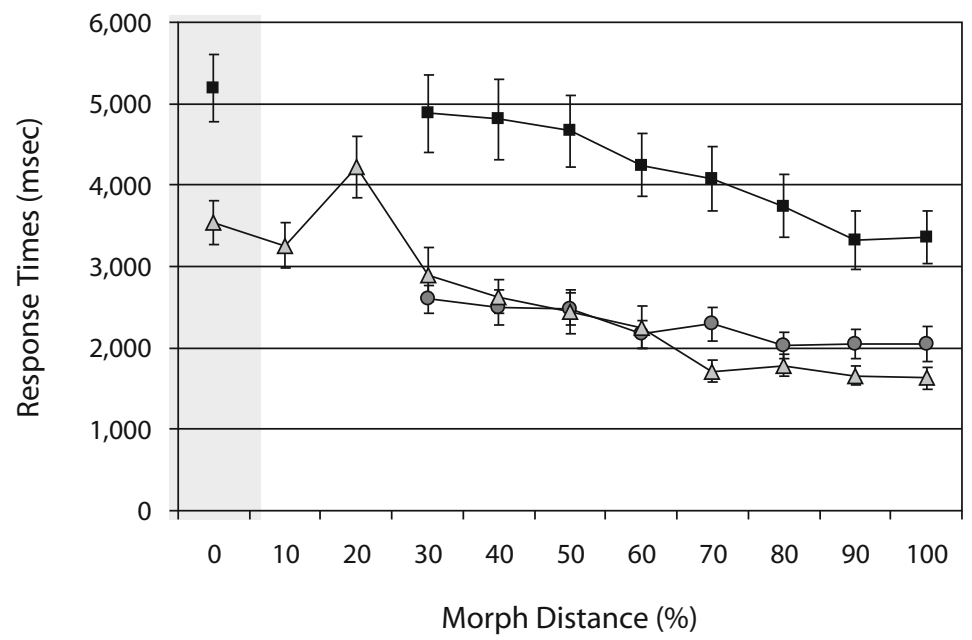

Figure 3. Psychophysical data for Experiment 1. (A) Performance, shown as the mean percentage of "different" ratings, equivalent to correct responses for all morph distance levels except $0 \%$ ("same pairs," shaded columns). Note that in the feminine task, no such "same pairs" were presented, and that in the sex and feminine tasks, no face pairs at the morph distance $10 \%$ or $20 \%$ were used. There were as many same trials as different trials in the sex and the identity tasks. $N=\mathbf{2 0}$ (sex and feminine tasks), and $N=18$ (identity task). Error bars represent $S E M$ s. (B) Response times (in milliseconds).

The significance level threshold was set to $p=.05$. Morph distance (i.e., the difference in morph percentage between the two faces shown in a trial; see Figure 1) was used as a within-subjects factor, and sex of participants was used as a between-subjects factor. To test whether the relationship between the physical manipulation (i.e., a linear operation) and the perception of our stimuli was monotonic (as was hypothesized), a linear trend analysis across consecutive distance levels was calculated (i.e., level $30 \%-100 \%$ for the sex and feminine tasks, level $10 \%-100 \%$ for the identity task). The distance level $0 \%$ was not taken into account for the linear trend, because it represents a differ- ent kind of stimuli (identical faces) and expected response ("same").

As a "correct" trial in the sex and the identity tasks, we counted a "same" response for morph distance level 0 , and a "different" response for all other levels $(10 \%-100 \%)$. In the feminine task, a response was correct if observers indicated the one face of a pair that was closer to the feminine endpoint face (see Figure 1).

\section{Experiment 1}

Performance. Results for all tasks are shown in Figure $3 \mathrm{~A}$. The ANOVAs revealed an effect of morph distance 
for all tasks [sex, $F(8,144)=30.70$; identity, $F(10,160)=$ 56.84; feminine, $F(7,126)=6.47$; all $p$ s $<.0001]$ but no effect of sex of participants. In all tasks, a linear trend along consecutive morph distance levels was found [sex, $F(1,18)=195.48$; identity, $F(1,16)=159.84$; feminine, $F(1,18)=18.63 ;$ all $p \mathrm{~s}<.0001]$.

RTs. Data are shown in Figure 3B. There was an effect of morph distance for the three tasks [sex, $F(8,128)=$ 7.37; identity, $F(10,70)=11.72$; feminine, $F(7,126)=$ 4.53; all $p \mathrm{~s}<.0001$ ], but no effect of sex of participants. In all three tasks, a linear trend of RT along consecutive morph distance levels was found [sex, $F(1,16)=44.24$; identity, $F(1,7)=8.56$; feminine, $F(1,18)=14.00$; all $p \mathrm{~s}<.005]$.

Wilcoxon signed rank tests revealed significant differences for both psychophysical measures between all tasks (RT: sex-feminine, $Z=-10.094$; identity-feminine, $Z=$ -4.535 ; identity-sex, $Z=-8.982$. ACC: sex-feminine, $Z=-8.821$; identity-feminine, $Z=-8.088$; identitysex, $Z=-5.0640$; all $p$ s $<.0001)$.

To summarize, in all three tasks male and female observers were better and faster when the two faces on the screen were at a large morph distance, and their performance became worse as well as slower with decreasing morph distance. Thus, the similarity of the faces of a pair was indeed monotonically related to the linear manipulation in physical space, allowing us to directly relate the fixation measures to this factor as well (see below). Performance was best in the feminine task; observers were less good in the identity task and worst as well as much slower in the sex task. It seems worth mentioning again that the face stimuli were manipulated in exactly the same way in both the sex and the feminine tasks.

\section{Experiment 2}

In Experiment 1, all participants performed the feminine task last. Experiment 2 was designed to test whether task order might influence performance and thus explain superior performance in the feminine task. All participants performed only the feminine task in Experiment 2.

The mean percentage of correct responses to all stimuli pairs across participants is shown in Figure 4 as "Fem ${ }^{1 s t}$." The performance data of the sex task in Experiment 1 is split into two groups: "Sex 1st" and "Sex ${ }^{2 n d, " ~ w h i c h ~ r e p r e s e n t ~ d a t a ~}$ of those participants who had performed the sex task first and second (i.e., after the identity task), respectively. Similarly, "Fem ${ }^{2 n d "}$ in Figure 4 shows the performance for the feminine task in Experiment 1. Even though there is some within-experiment training effect, because participants' performance for the feminine task is worse in Experiment 2 than in Experiment 1, it clearly surpasses performance for the sex task [Wilcoxon: Fem ${ }^{1 s t}$ sex (both groups), $p=.000$, $Z=-8.839]$. In consequence, since the role of training has been refuted, the question remains whether this striking difference in performance between the sex and the feminine task is paralleled and might be elucidated by distinctive eyemovement patterns, analogous, for example, to what has been shown for differing facial information processing in same-race and other-race faces (see the introduction).

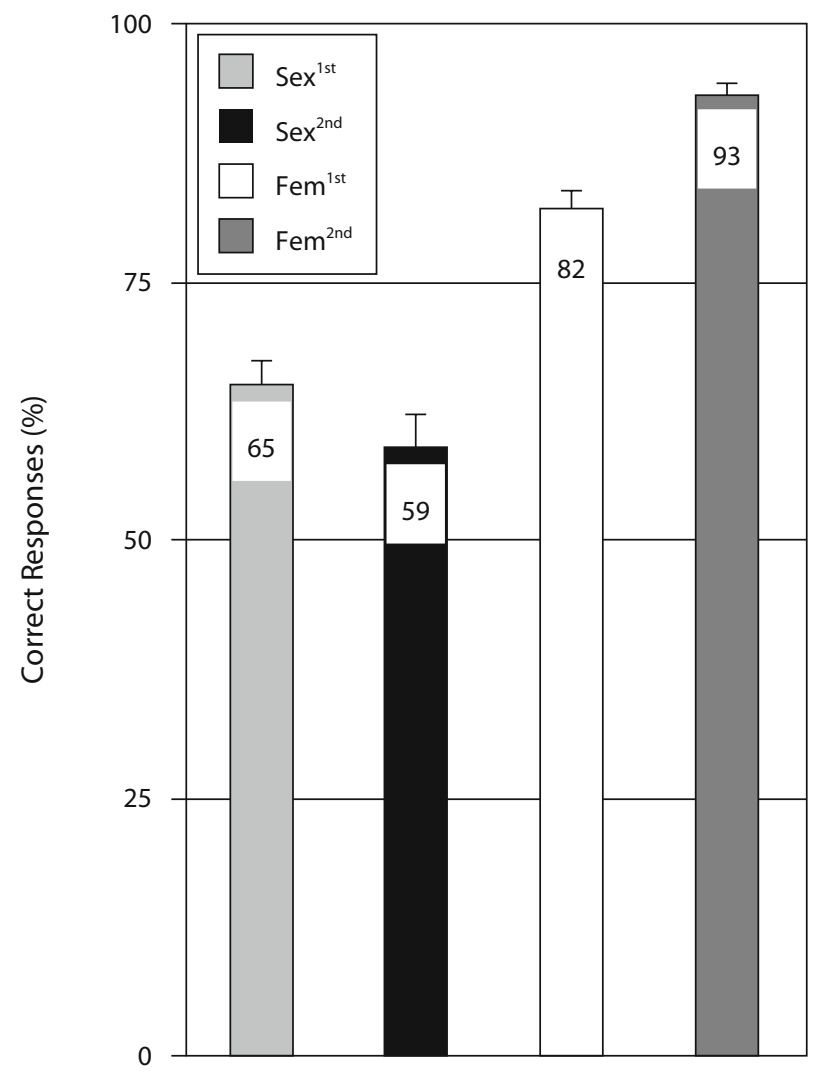

Figure 4. Control for training effects. Mean number of correct responses for participants who did the sex task first in Experiment 1 (Sex ${ }^{1 s t}, N=12,6$ male, 6 female), or after the identity task (Sex ${ }^{2 n d}, N=8,5$ male, 3 female). Fem 1 st shows accuracy for participants who did only the feminine task (Experiment 2, $N=17$, 8 male, 9 female), Fem ${ }^{2 n d}$ shows accuracy for those who did the feminine task at the end of Experiment $1(N=20,10$ male, 10 female). Error bars represent SEMs.

\section{Eyetracking Data Analysis}

Repeated measures ANOVAs were conducted for each task separately. The significance level threshold for all statistical analyses was set to $p=.05$. For some global measures, tasks were compared with the nonparametric Wilcoxon signed rank test to account for different task designs.

\section{Single Fixation Duration in Direct Comparison}

Longer fixations imply that the user is spending more time interpreting or relating the observed visual stimulus to internalized representations (Goldberg \& Kotval, 1999). Buswell (1935) found that observers made longer fixations with increasing task difficulty. RM ANOVAs with morph distance as a within-subjects factor and sex of participants as a between-subjects factor revealed no significant effects, since fixation duration did not vary with difficulty. This result is in accordance with our assumption that in a comparison task, there is no need to internally relate stimulus and representation, since all of the information necessary to solve the task is visually available and can be inspected as often as necessary. The mean 
A

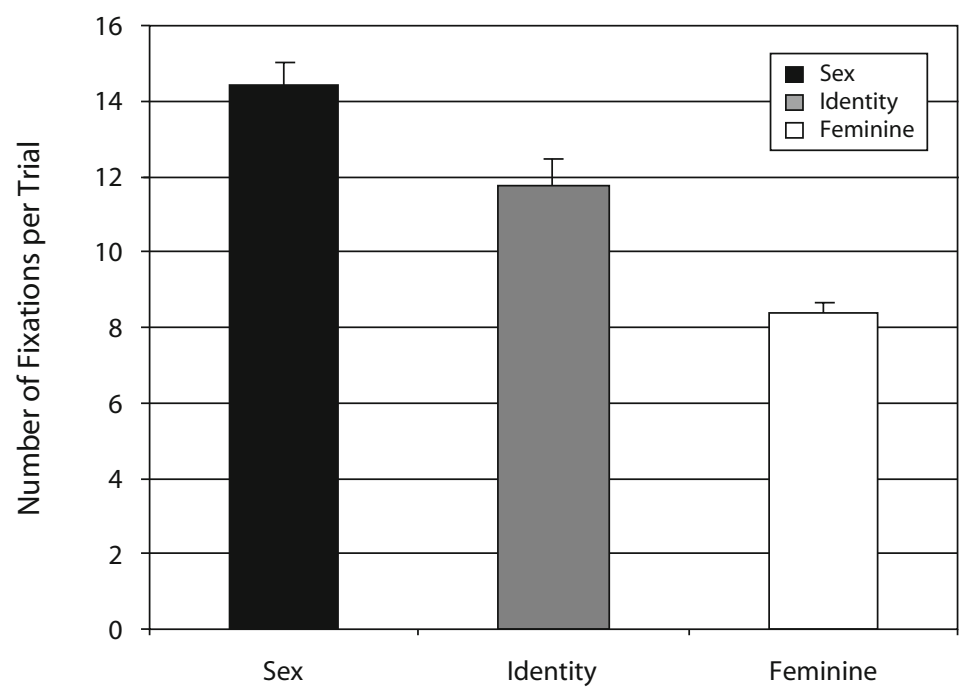

B

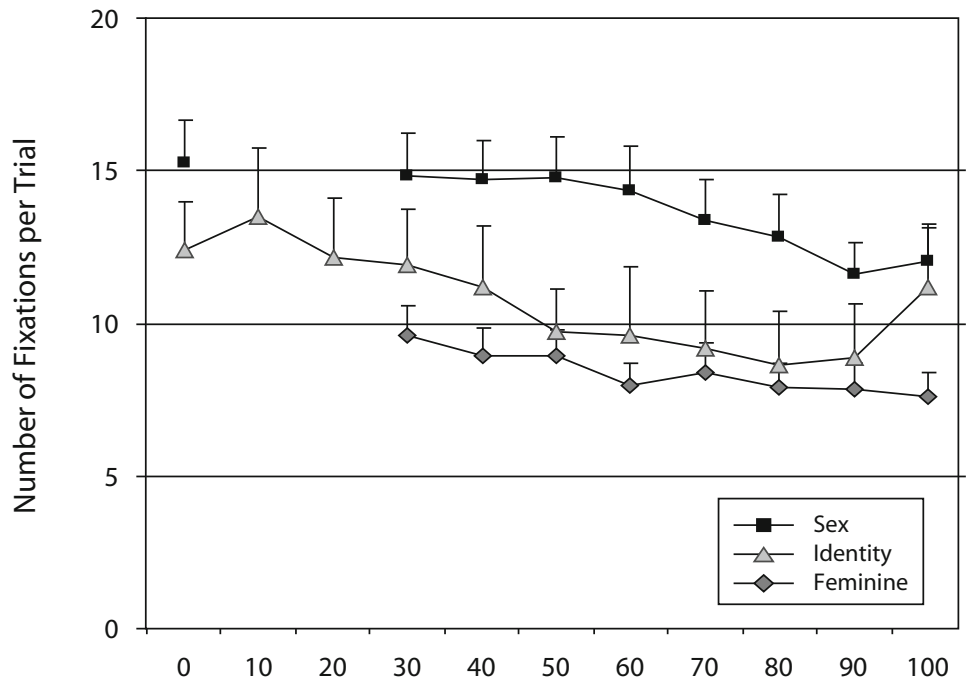

Figure 5. Mean number of fixations for each task on average (A) and across morph levels (B). Error bars represent $S E M$ s. $N=20$ (sex and feminine tasks), and $N=18$ (identity task).

length of a single fixation was not significantly different in the three tasks, which ranged from 351 msec (identity task, $S E M=22$ ) to $382 \mathrm{msec}$ (feminine task, $S E M=24$; sex task, $M=367 \mathrm{msec}, S E M=23$ ). Since single-fixation duration did not vary across AOIs either, the distributions of the numbers of fixations and of cumulative fixation time in the different AOIs are highly correlated and thus redundant. We will therefore present only results related to the number of fixations.

\section{Overall Number of Fixations per Task}

Figure 5 shows the overall fixation number per trial for each task. Overall, observers made the most fixations (and thus spent most time fixating) in the sex task, fol- lowed by the identity task and then the feminine task (Wilcoxon signed rank: sex-feminine, $Z=-8.452$; identityfeminine, $Z=-3.033$; identity-sex, $Z=-5.862$; all $p$ s $<.005)$. These findings correlate with the RTs for the tasks (see above), showing that the worse and the slower the performance, the more fixations that were made (and the more time that was spent looking at the faces).

That the relationship among ACC, RT, and fixation time was not caused by different levels of observers' attention is indicated by similar values of search efficiency in the three tasks. It is calculated as the number of fixations on both faces divided by the number of all fixations made on the screen during a trial (Goldberg \& Kotval, 1999). A value of 1 would mean that no fixation was made out- 
Table 1

Repeated Measures ANOVA on Fixation Numbers With the Factors Face, Area of Interest (AOI), Morph Distance (Within Subjects), and Sex of Participant (Between Subjects)

\begin{tabular}{|c|c|c|c|}
\hline \multirow[b]{2}{*}{ Factor } & \multicolumn{3}{|c|}{ Task } \\
\hline & Sex & Identity & Feminine \\
\hline Face (left/right) & n.s. & n.s. & $\begin{array}{l}F(1,18)=10.123 \\
p=.005\end{array}$ \\
\hline AOI (10 areas per face) & $\begin{array}{l}F(9,162)=21.329 \\
p=.000\end{array}$ & $\begin{array}{l}F(9,144)=10.155 \\
p=.000\end{array}$ & $\begin{array}{l}F(9,162)=14.125 \\
p=.000\end{array}$ \\
\hline $\begin{array}{l}\text { Morph distance } \\
\quad \text { (similarity of faces) }\end{array}$ & $\begin{array}{l}F(8,144)=12.383 \\
p=.000\end{array}$ & $\begin{array}{l}F(10,160)=9.914 \\
p=.000\end{array}$ & $\begin{array}{l}F(7,126)=7.207 \\
p=.000\end{array}$ \\
\hline Face $\times$ AOI & $\begin{array}{l}F(9,162)=21.107 \\
p=.000\end{array}$ & $\begin{array}{l}F(9,144)=18.590 \\
p=.000\end{array}$ & $\begin{array}{l}F(9,162)=19.231 \\
p=.000\end{array}$ \\
\hline AOI $\times$ morph distance & $\begin{array}{l}F(72,1296)=2.532 \\
p=.011\end{array}$ & $\begin{array}{l}F(90,1440)=2.542 \\
p=.030\end{array}$ & $\begin{array}{l}F(63,1134)=3.282 \\
p=.001\end{array}$ \\
\hline AOI $\times$ sex of participant & $\begin{array}{l}F(9,162)=2.897 \\
p=.034\end{array}$ & n.s. & $\begin{array}{l}F(9,162)=2.616 \\
p=.008\end{array}$ \\
\hline $\begin{array}{l}\text { Linear trend along } \\
\text { morph distance }\end{array}$ & $\begin{array}{l}F(1,19)=37.594 \\
p=.000\end{array}$ & $\begin{array}{l}F(1,17)=34.620 \\
p=.000\end{array}$ & $\begin{array}{l}F(1,19)=20.578 \\
p=.000\end{array}$ \\
\hline
\end{tabular}

side the face stimuli (which covered about $28^{\circ} \times 20^{\circ}$ of a $40^{\circ} \times 30^{\circ}$ screen), whereas smaller ratios indicate lower efficiency. Search efficiency was highest for the sex (.92, $S E M=.07)$ task and slightly lower for the identity $(.89$, $S E M=.06)$ and feminine $(.86, S E M=.04)$ tasks, thus following a trend that was the inverse of that for ACC.

\section{General Analysis of Eye-Movement Patterns}

Repeated measures ANOVAs with the within-subjects factors of face (two levels, right or left on the screen), AOI (10 levels per face), and morph distance ( 9 levels for sex, 11 levels for identity, 8 for feminine) were conducted on numbers of fixations. The between-subjects factor was sex of participants. See Table 1 for complete $F$ and $p$ values. In the following sections, the results will be described with respect to the three main questions that were raised in the introduction: (1) Which facial features are considered essential for solving the tasks? (2) How do observers compare the two faces? (3) Do the results differ for male and female participants?

(1) Which features do play a role? Does the task make a difference? The numerous AOIs on the faces received unequal numbers of fixations, resulting in a significant effect of AOI on fixations for all tasks. Figure 6 shows the overall fixation pattern in AOIs (collapsed across features and both faces) for the pilot study (Figure 6A) and for Experiment 1 (Figure 6B), demonstrating that there was no effect of the differing time limits on looking behavior: Even though only a few participants performed this pilot experiment, the eye movements to the AOIs already show a general pattern that corresponds to the one in the main experiment (note especially the eye and nose regions). See the figure legend for the numbers of participants. In the following, we will refer only to the data of Experiment 1 (Figure 6B).

Participants fixated most often and the longest on the eyes and the nose in all tasks, followed by the mouth in the sex task and the cheeks in the identity and feminine tasks.
The eyes were more often looked at in the sex task (on average, $36.8 \%$ of all fixations in a trial) than in the identity $(31.6 \%)$ and the feminine (28.8\% of all fixations) tasks. The eyes received significantly more fixations than did all other AOIs, except for the nose in the sex task only ( $p<$ .003 , Bonferroni-corrected post hoc pairwise comparisons). The nose was looked at most in the feminine task (32.0\% of all fixations vs. $26.3 \%$ in the sex and $24.5 \%$ in the identity tasks). Unlike in the other tasks, in the feminine task, the nose was looked at slightly more often than were the eyes (although the differences between the eyes and nose were never significant). Wilcoxon signed ranks tests between tasks revealed no significant difference for fixations to the eyes or the nose region.

Observers spent more fixations on the mouth area in both same-different tasks than in the feminine task (only $7.5 \%$, vs. $13.44 \%$ and $14.45 \%$; Wilcoxon: feminine-sex, $Z=-1.904, p=.041$; feminine-identity, $Z=-2.330$, $p=.020)$. The cheeks were looked at less often in the sex task (11.24\%) than in the other two tasks (identity, $17.99 \%$; feminine, $19.44 \%$ of all fixations; Wilcoxon: n.s.). Numbers of fixations to the face outline were highest in the feminine task and lowest in the sex task (Wilcoxon: feminine-sex, $Z=-3.883, p=.000$; identity-sex, $Z=-2.199, p=.028)$. In sum, our first eye-movementrelated questions can be answered positively: Some facial features are effectively considered more diagnostic than others, and which feature is looked at more often depends on the task.

Where to look first? We analyzed the data for each task with respect to first fixations and subsequent fixations; the results for first fixations are shown in Figure 7A as the percent of first fixations in each trial averaged across participants. Since the results for subsequent fixations practically do not differ from the results for all fixations, we will not present additional figures for subsequent fixations. When we collapse the data for first fixations across corresponding AOIs and both faces (not shown), a pattern 
A

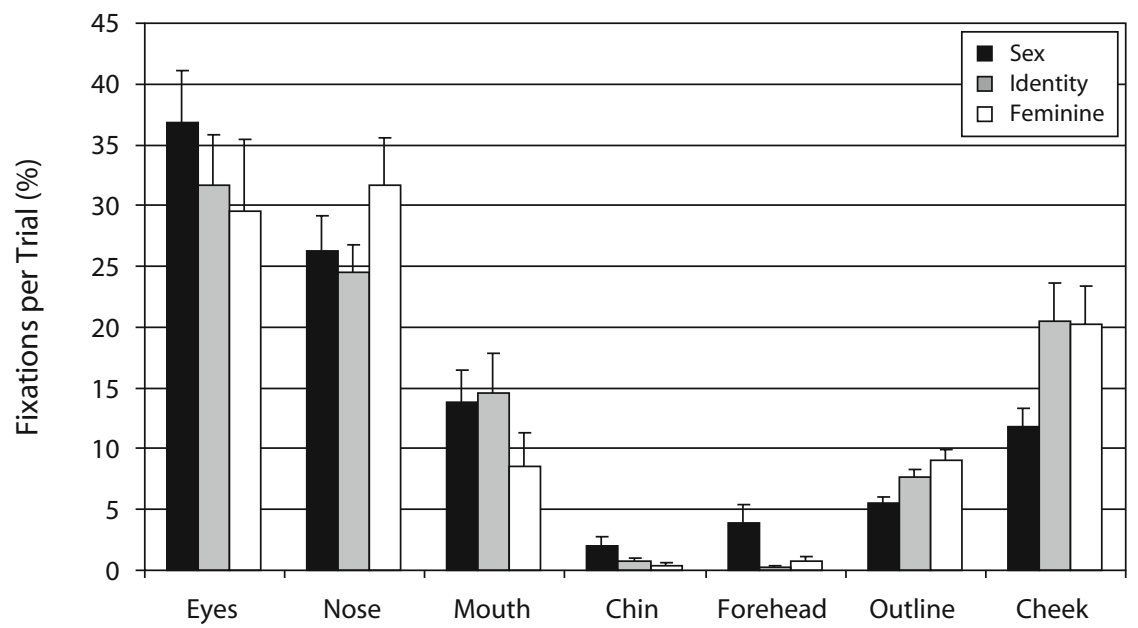

B

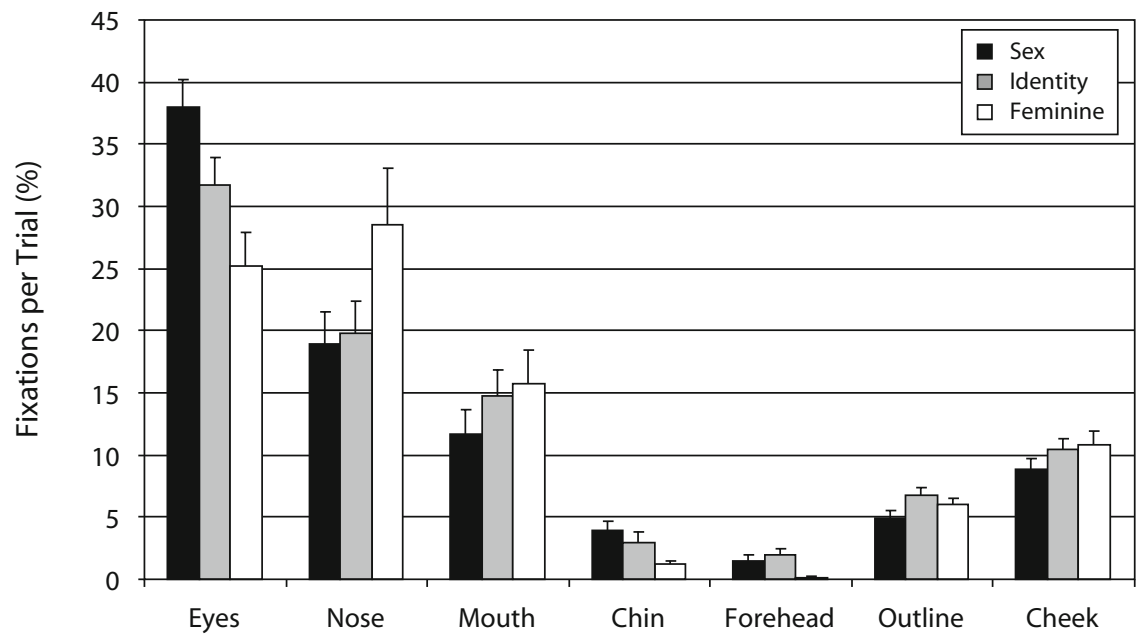

Figure 6. Number of fixations in areas of interest. (A) Pilot experiment without time restriction (sex task, $N=11$; identity task, $N=10$; feminine task, $N=8$ ). (B) Experiment 1 (sex and feminine tasks, $N=20$; identity task, $N=18$ ). Mean percentages of fixations per trial are shown for each task. Fixations are collapsed over features that were represented by more than one area of interest; for example, "eyes" represents both eyes of both faces on the screen. Error bars represent SEMs.

appears that is similar to that of the data for subsequent and/or all fixations (see Figure 6 for results calculated over all fixations). For a closer look at the data, in Figure 7, we present numbers of fixations for each single AOI for first fixations (7A) and all fixations (7B). The most striking finding here is that observers' first fixation fell on the nose region of the left face much more often in the feminine task than in the other two tasks (Wilcoxon signed ranks: feminine-sex, $Z=-3.454, p=.001$; feminineidentity, $Z=-3.610, p=.000$ ), whereas there was no difference between the sex and the identity tasks. Even though there are other minor variances between tasks concerning the first fixation in a trial, this most substantial difference for the nose region is in line with the results that we have reported so far about more fixations on aver- age on this region in the feminine task than in the other two tasks.

What if the task gets more difficult? The effect of morph distance was significant for all tasks, as was the interaction of AOI and morph distance. There was a linear trend of numbers of fixations along consecutive morph distance levels in all tasks, which was due to the continuously decreasing numbers of fixations with decreasing difficulty (see Figure 5B). This result is again in accordance with the relation we found between task performance and fixation time (see Figure 3). Thus, in general, the more difficult a trial or the whole task was, the more fixations the participants made.

When we looked closer at the linear relationship between numbers of fixations and difficulty, we found that 

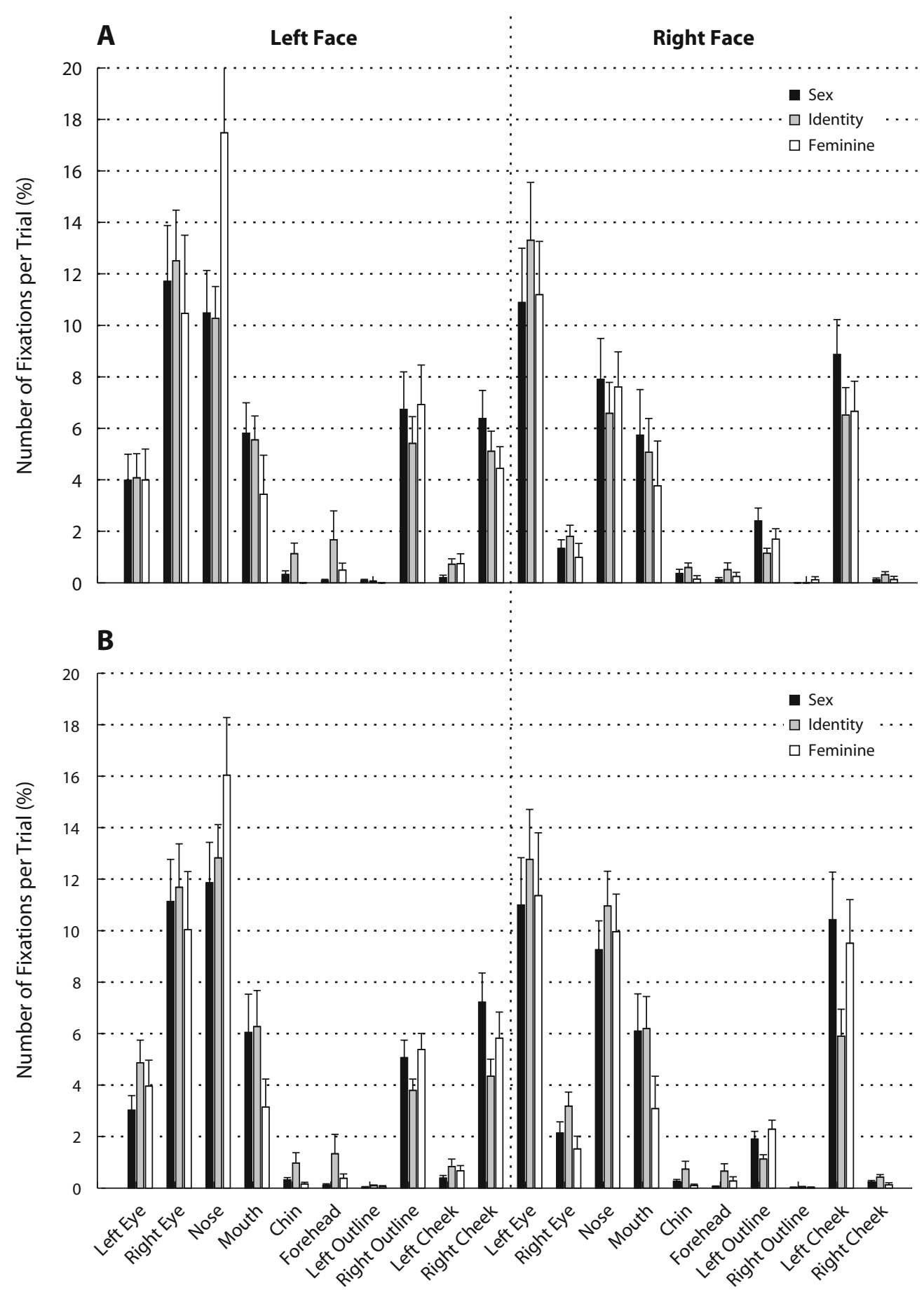

Figure 7. Mean percentages of the first fixation (A) and of all fixations (B) to all single areas of interest in each trial (sex and feminine tasks, $N=20$; identity task, $N=18$ ). Error bars represent $S E M s$.

with decreasing morph distance between the two faces in a trial (i.e., increasing similarity), more fixations were made only on a few AOIs. For each AOI, we calculated one-way repeated measures ANOVAs including linear trend analyses for the numbers of fixations along consecutive morph distance levels. AOIs showing a significant effect for the ANOVA and the linear trend (and thus contributing to the interaction of AOIs and morph distance) are shown in Fig- ure 8. The "inner" eye regions (i.e., the parts of the eyes closer to the middle of the screen), the nose, and the mouth of the left face show this morph distance effect in the sex task (all $p \mathrm{~s}<.04$ for the linear trend). In the identity task, the effect is found as well in the inner-eye regions, the nose, and both mouth regions, and additionally in the left outline and the left cheek of the right face (all $p \mathrm{~s}<.01$ ). In the feminine task, only the numbers of fixations on the 

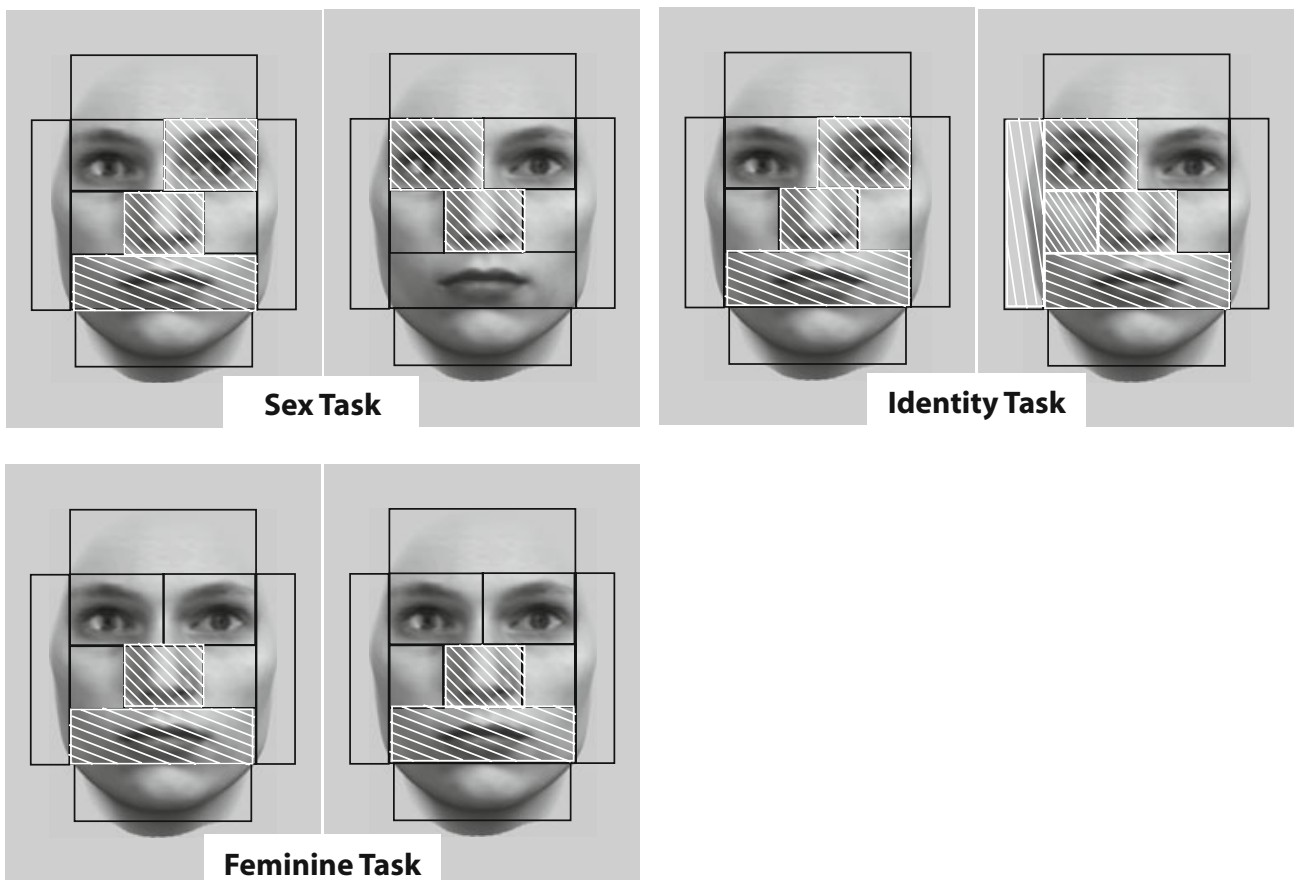

Figure 8. Interaction of areas of interest (AOI) and morph distance. Only in a few AOIs did the number of fixations increase significantly with increasing similarity between the faces in a trial. Repeated measures ANOVAs and linear trend analyses along morph distance levels were conducted within each AOI separately. The AOIs in which effects of both analyses were significant are highlighted for each task.

nose and mouth area significantly increase with decreasing morph distance (all $p \mathrm{~s}<.012$ ).

It appears from these results that when task difficulty increased due to increasing face similarity (i.e., decreasing morph distance) within a task, observers made more fixations only to a few AOIs and did not distribute them evenly over the faces. Those AOIs receiving increased scrutiny differed slightly, depending on the task. The most obvious difference is that although the eyes were the mostlooked-at facial features in the whole experiment, they were not considered informative in the feminine task, in which observers were facing higher task difficulty.

(2) How are two faces compared? The ANOVA revealed a significant effect of face (left or right of a pair) only for the feminine task; this was due to a higher number $(18.9 \%, S E M=1.9)$ of fixations per trial on the left face than on the right face $(12.72 \%, S E M=1.7)$, only in this task. Consistently, of the first fixations in the feminine task (see Figure 7), 41.6\% $(S E M=1.6)$ fell on the left face, but only $32.5 \%(S E M=1.2)$ fell on the right face (this discrepancy was mainly due to the very high percentage of first fixations to the left nose), whereas this difference was much smaller (sex task: $37.9 \%$ left, $S E M=0.9$; $35.9 \%$ right, $S E M=1.4$ ) or nonexistent (identity task: $37.9 \%$ left, $S E M=1.1 ; 37.8 \%$ right, $S E M=1.3$ ) in the other two tasks.

The ratio of transitions between the two faces was calculated for each task by counting the gaze transitions from one face to the other in each trial and dividing this number by the sum of all fixations made in the trial. The maximum ratio of 1 indicates that gaze was moved from one face to the other after each single fixation. This measure was assessed to give insight into how participants compared the two faces in general. No significant differences or meaningful trends were found within tasks for this measure. On average, participants made approximately two fixations on one face before moving their gaze to the other face in all tasks [mean ratio of transition was .44 in the sex $(S E M=.00)$ and the feminine $(S E M=.01)$ tasks and .47 in the identity task $(S E M=.01)]$.

Is the left face half more informative? The interaction of $\mathrm{AOI}$ and face was significant for all tasks, suggesting that observers directed their attention to different AOIs on the two faces in a trial. Figure 9 reveals differences between numbers of fixations on AOIs that exist on both sides of each face-for example, the eyes. For further analyses, we separated the AOIs into two categories. The mouth, chin, and forehead belong to the "central" AOIs, since they are present only once in a face. Eyes, cheeks, and facial outline exist as left and right AOIs on each face and thus represent the "bilateral features" (compare Figure 2 with Figure 9).

The Bonferroni-corrected post hoc pairwise comparisons that were conducted between all 20 AOIs subsequent to the general RM ANOVA revealed that the numbers of fixations spent on the "central features" did not differ between the left and right faces in a trial, except for the nose in the feminine task $(p<.05)$; the nose of the left face was looked at more often and longer than was the nose of the right face (6.18\% of fixations more on left nose). Thus, ap- 
Bilateral Features

\section{Central Features}

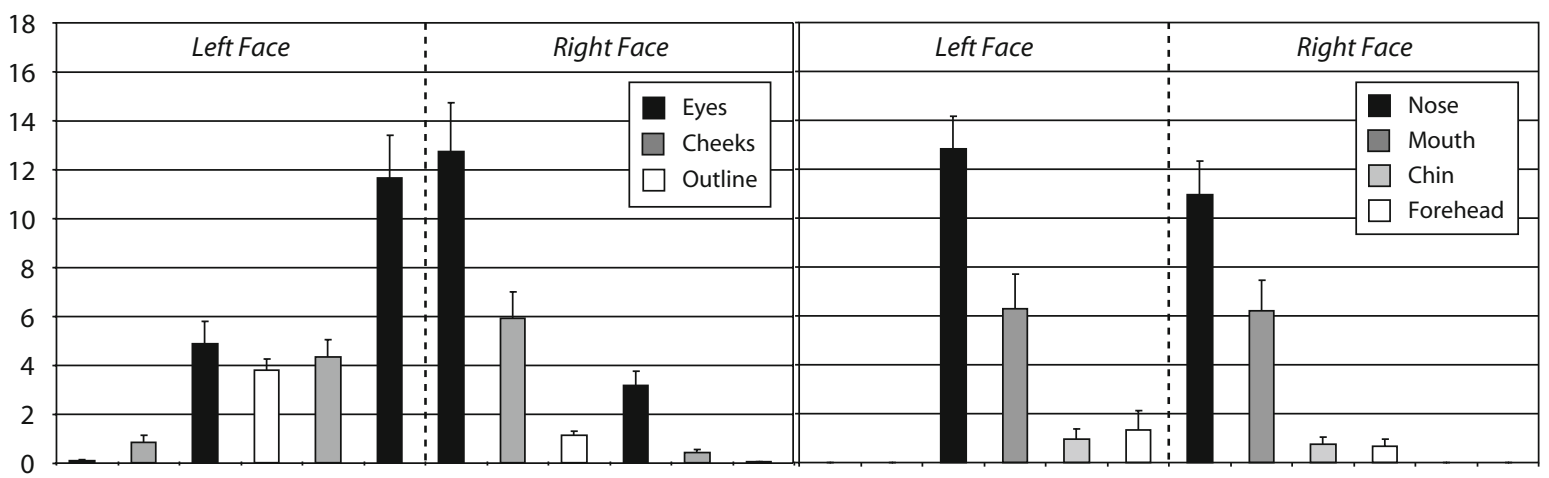

Sex Task

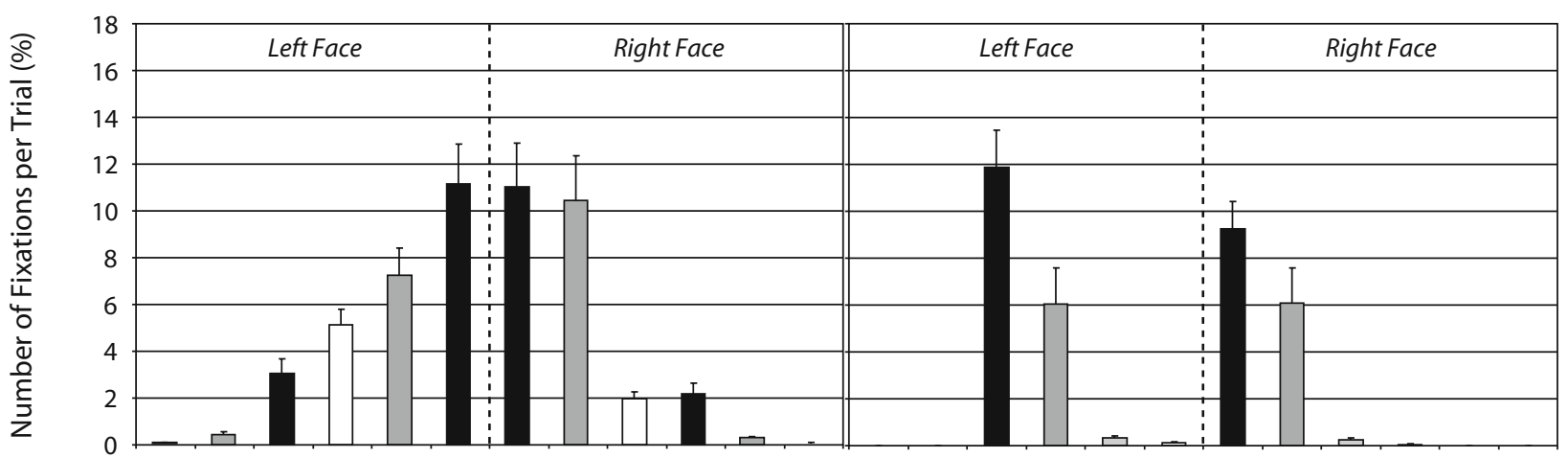

Identity Task

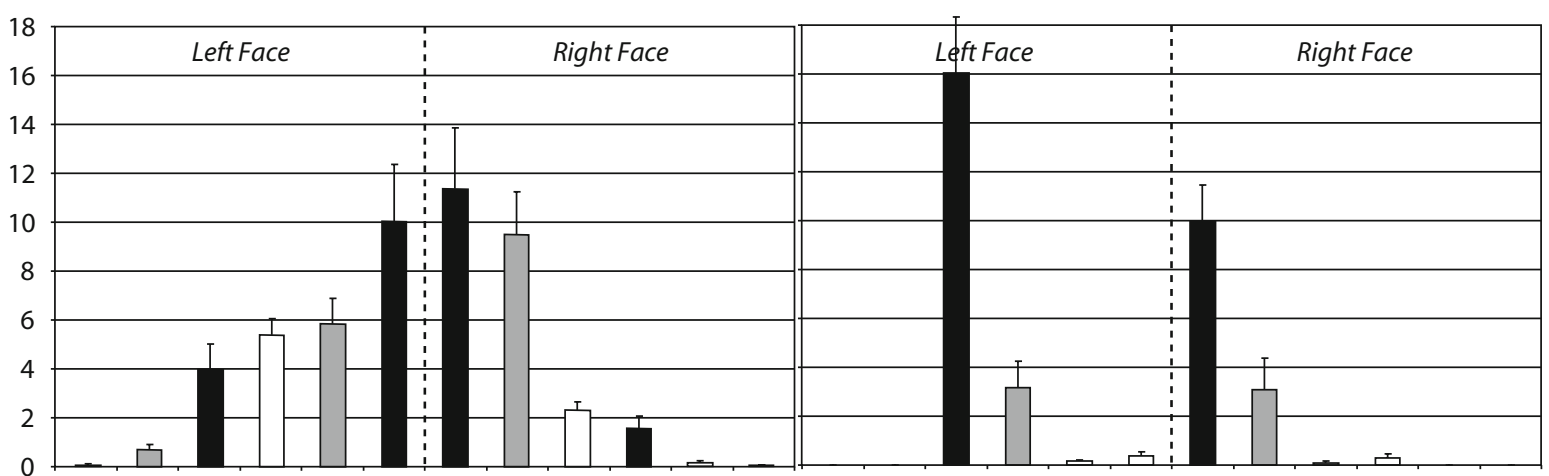

Feminine Task

Figure 9. Asymmetries in viewing patterns. Areas of interest are grouped as "bilateral" features (appearing twice on a face, left) and "central" features (only one per face, right). The numbers of fixations on central features do not differ between the left and right faces, except for the nose in the feminine task. The numbers of fixations on bilateral features differ significantly between faces and between both regions on one face. Error bars represent $S E M$ s.

parently fixations on the nose were responsible for the main effect of the factor face in the feminine task (see above).

In contrast, we found significant differences for numbers of fixations on the most "bilateral features." In the sex and identity tasks, within each face, fixations on both eye areas differed, whereas between faces, fixations on the left eyes of both faces differed from each other. The same effect was found with fixations on the right eyes of both faces (all $p \mathrm{~s}<.02$ ). In the feminine task, in which the eyes received fewer fixations in relation to the other features (see above), the same differences in numbers of fixations were observed but did not reach significance (see Figure 9). For the other bilateral features (left and right cheeks, left and right outlines), the same pattern was found as that for the eyes, and comparisons between and within faces were significant in all tasks (all $p \mathrm{~s}<.032$ ).

To conclude from these results and the graphs shown in Figure 9, in all tasks, the bilateral features on the inner 
halves of the faces (i.e., those closer to the screen center) were looked at more often than were the features of the outer halves of the faces.

Scan-path strategies: Is there a stereotyped pattern? Viewing behavior can be described as a temporal sequence of successive fixations in different AOIs. We investigated whether the observed sequences show similarities across morph distance levels in each task. To examine the global predictability of scanning - that is, how much the whole scanning sequence of the faces of one trial resembled that of another trial-we did a string-edit comparison of all trial sequences within each task. To this effect, we computed the Levenshtein distance, a measure borrowed from DNA sequence analysis to calculate the "editing cost" (how many elements need to be changed) of transforming one sequence into another (Gilleland, n.d.).

We found that the average Levenshtein distance between all sequences divided by the average length (i.e., the number of fixations) of the two sequences being compared varied only slightly (yet in accordance with the difficulty of the tasks), as follows: It was highest for the sex task (0.41), slightly lower for the identity task $(0.40)$, and lowest for the feminine task $(0.36)$. Thus, viewing patterns were slightly more diverse in the more difficult tasks. The results are more meaningful for the variation of sequences within each task: Linear trend analyses across consecutive morph distance levels showed that the Levenshtein distance significantly increased with difficulty (i.e., decreasing morph distance) within all tasks $(F>21.00, p<$ .001 , for all tasks). In addition to the similarity between scan paths, we then searched for distinct patterns in the fixation sequences, as follows.

Do we look again? First, immediately repeated fixations on the same region during a trial were considered an indicator of local feature-based processing as opposed to the generation of a global face percept (Barton et al., 2006). The results do not contribute any new information (neither significant effects nor meaningful trends in any of the factors we investigated); it is only worth noting that repeated fixations happened very rarely throughout the whole experiment. That is, in general, after fixating on one AOI, observers' eye gaze moved to another AOI.

Which eyes are compared? More interesting results were obtained when we scanned the eye-movement sequences more precisely for successive eye fixations (i.e., successive fixations from one eye region to another eye region on the same or other face), since eyes are the bilateral facial features that were looked at most frequently in general throughout the experiment. We calculated first the ratio of all successive eye fixations (see Figure 10) - that is, the number of fixations on one eye region followed by one to any other eye region, in relation to half the number of all eye fixations in a trial (i.e., all possible repetitive fixations). No difference between tasks was found for this measure.

Then, in the same way, the ratio of "symmetrical" successive fixations was calculated (i.e., from the left/right eye of the left face to the left/right eye of the right face and vice versa), as was the ratio of "asymmetrical" successive fixations - for example, from the right (inner) eye on the left face to the left (inner) eye on the right face. As is

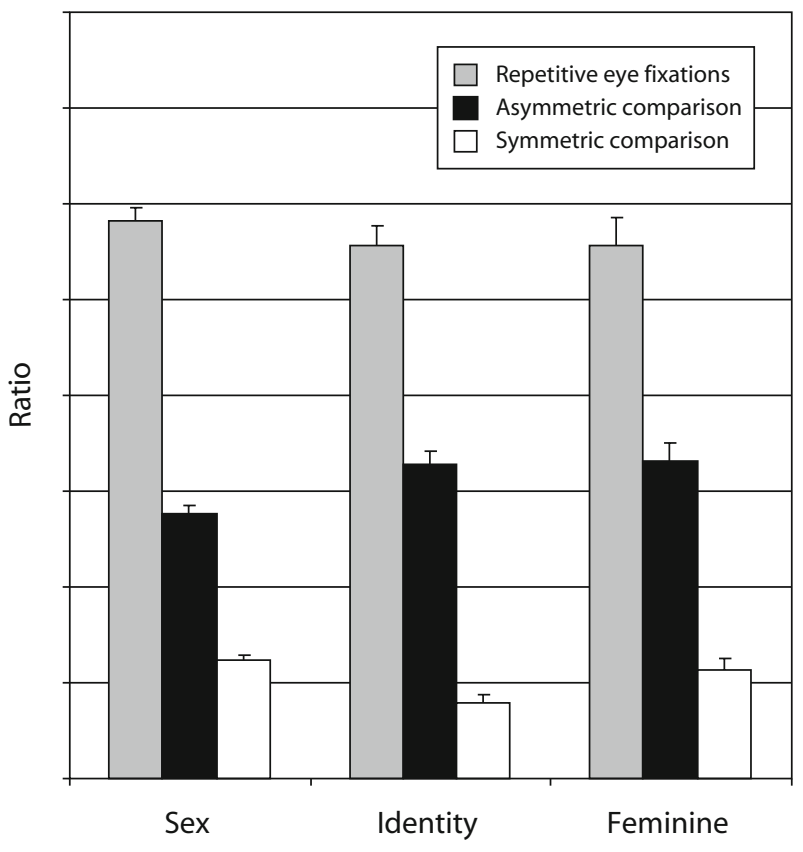

Figure 10. Gray bars show the mean number of successively repeated fixations to any eye area per trial divided by half the number of fixations made in that trial- that is, the number of possible repetitive fixations. The same ratio was calculated for "asymmetrical comparisons" between the eye regions of both faces (black bars) and "symmetric comparisons" (white bars). Error bars represent $S E M$ s.

clearly visible in Figure 10, observers compared the eyes of a face pair asymmetrically most of the time, moving their gaze from inner eye to inner eye or (less often) from outer eye to outer eye. This clarifies the fixation pattern shown in Figure 9, where the features/eyes closer to the center of the screen were looked at more often than were the outer features/eyes of both faces.

(3) Does observer's sex make a difference? No main effect of sex of participants was found in any of the tasks, but an AOI $\times$ sex of participants interaction was present in the sex and the feminine tasks. Figure 11 shows the mean percentage of fixations on AOIs (collapsed across bilateral features and both faces) for male and female participants separately for these two tasks. The differences between male and female observers' results are the following: Female participants made, on average, more fixations to the eyes of the faces than did male participants. Independent-samples $t$ tests that were conducted to compare fixating behavior of male and female observers to each AOI revealed significant differences for the eye regions in both tasks $(p<.025)$. Additionally, male participants made, on average, more fixations to the cheeks than did female participants, although this difference between participants reached significance only in the feminine task $(p=.048)$.

\section{DISCUSSION}

\section{Performance}

The two main findings for the performance data are the following: First, unsurprisingly, participants were bet- 


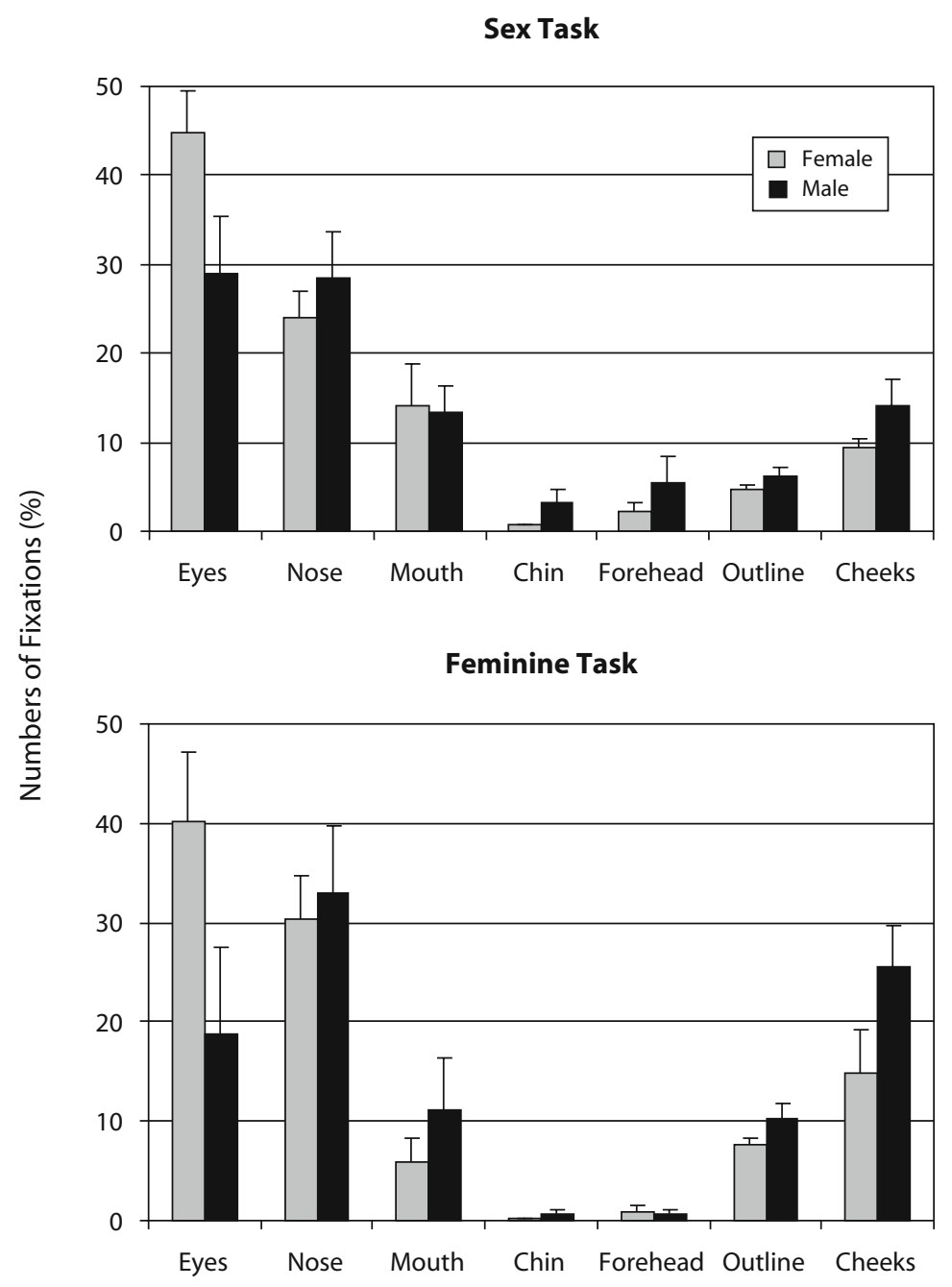

Figure 11. Mean percentages of fixations per trial per area of interest, shown for male and female participants separately. Features are collapsed across bilateral features and both faces. $N=20$ in both tasks.

ter and faster the more different the faces in the test pair were. Second, there were large differences in performance between the three tasks. We will review these two findings before considering the data on eye movements.

In all tasks, test pairs of linearly increasing similarity increased task difficulty in a monotonically related way. Although the fact that more similar stimuli are more difficult to discriminate is unsurprising, it is worth noting that no sudden changes in performance were observed, since a linear relationship between performance (or RTs) and morph distance was established. These findings are in agreement with those of previous studies (Angeli, Davidoff, \& Valentine, 2001; Bülthoff \& Newell, 2004) on categorical perception that failed to report a sudden change in performance - a hallmark of categorical effect - in unfamiliar typical faces when the sex or identity of the faces was manipulated.

Performance varied considerably between tasks; the sex task was clearly more difficult than the identity task, whereas the feminine task was the easiest to solve. In the identity and sex tasks, participants made the same type of judgment (same or different), with face stimuli manipulated along two different dimensions. The results show that for corresponding morph distance, performance was always better and faster for face pairs in the identity task. These findings are in accordance with a previous study by Bülthoff and Newell (2004), who reported discrimination between faces differing in sex to be more difficult than that for faces differing in identity. One might assume that performance in the identity task was better simply because identity morphs differ perceptually more from each other than do sex morphs. However, this assumption is not supported by the other results of the experiment, since performance - in comparison with the results of the identity task - was better for the feminine task and much worse for the sex task (although in both of these tasks, faces underwent identical sex manipulations). In low-level psychophysics, it has been reported repeatedly that performance in a $2 \mathrm{AFC}$ task is in general somewhat higher than that in a same-different task (see, e.g., Chen 
\& Macmillan, 1990; Creelman \& Macmillan, 1979; and Macmillan, Goldberg, \& Braida, 1988, all as cited in Macmillan \& Creelman, 1991). In our study, for face pairs at identical morph distance levels-for example, at a 30\% distance - participants were unable to tell that the faces differed (i.e., there were fewer than $30 \%$ correct responses for this level) in the sex task, but when they knew that the faces differed (in the 2AFC-feminine task), they could correctly judge which one was more feminine in more than $80 \%$ of the cases. In our opinion, this difference in performance is too high to be a mere consequence of the "task factor" mentioned for low-level psychophysics. We think that another factor influencing observers' discrimination capacities might be differences in attention allocation caused by the instructions for the task at hand. Participants apparently were induced to look at the stimuli in a way that was adequate for allowing a correct answer in the feminine task but not in the sex task. Schyns and Oliva (1999) and Goffaux, Hault, Michel, Vuong, and Rossion (2005) have suggested that observers direct their attention to different spatial scales, depending on the facial information they are looking for. Our findings that observers' fixation behavior toward the face stimuli was indeed different in the two tasks (this will be discussed in the following section on eye movements) might be a reason for participants' huge performance differences, in agreement with Schyns and Oliva's suggestion.

Since the feminine task was always performed last by all participants, the question remained whether the surprisingly better performance observed for the feminine task might have resulted from implicit learning due to repeated exposure to the sex-morphed stimuli. The results of Experiment 2 (in which observers performed only the feminine task) show that this is not the case. Although observers' performance was reduced in Experiment 2 as compared with Experiment 1, it was significantly higher than performance in the sex task of Experiment 1, irrespective of whether participants had performed the sex task first or second in that experiment (see Figure 4). The effect cannot be due to particular face stimuli being accidentally easier to discriminate than others, since Experiment 2 was done with face stimuli that had been used in the sex or identity task of Experiment 1 before.

\section{Gaze Behavior}

Internal representations. In accordance with our suggestion that in a comparison task there is no need to internally relate the stimulus to any stored representation, we did not find variations in single fixation length related to task or difficulty. This corroborates the argument stated in the introduction - that is, that the facial features that are found to be essential to solving our comparison tasks pertain to a more general concept of identity and sex, rather than to a memory recall of specific faces necessary to solve the task at hand.

Diagnostic features. Our results confirm earlier findings reporting that not all facial features are equally important for face processing (see, e.g., Schyns et al., 2002). We have shown that the number of fixations made to dif- ferent AOIs varies strongly. This result cannot be related to the size of the areas since, for example, the largest region is the forehead (covering about $28 \%$ of the whole face), which received very few fixations on average, whereas the eyes were looked at most frequently, even though each of them represents only about $9 \%$ of a face surface. The most "important" regions regarding the fixations made to them were the eyes and the nose, in all tasks, followed by the mouth (sex task) and the cheeks (identity and feminine tasks). In contrast with previous studies (e.g., Pearson et al., 2003), the forehead was not extensively scanned in the present experiment. Presumably, since our face stimuli fade into the background and no hair line is visible, the forehead area does not provide much information about identity or sex.

Our results emphasize the task dependency of observers' intention (whether conscious or unconscious) to consider some facial features as more informative and worthy of attention than others. In both same-different tasks, the eyes were considered the most informative features (Figure 6). Nevertheless, the importance of the eyes over all other features (i.e., they were more often looked at) was highlighted even more in the sex task, as opposed to the identity task, in which (as we have shown) observers distributed more fixations over the whole faces. This finding about differing diagnostic features in the sex and the identity tasks is in accordance with recognition and sex classification studies mentioned earlier (Pearson et al., 2003; Schyns et al., 2002), and is also reflected in our analysis on increased numbers of fixations to only some specific AOIs when a task gets more difficult (Figure 8): Here again, in the identity task, more fixations are distributed to a higher number of regions besides the eyes and nose than is the case in the sex task. Our data corroborate what has been reported so far about diagnostic features in single-face studies involving face identification and sex classification of familiar faces. Since our study was specifically designed to eliminate the role of any task-related memory representations, we can now conclude from our results that these features are truly related to the "general concept" of identity and of the sex of faces.

In the feminine task, observers' viewing behavior differed slightly but consistently from their behavior in the two same-different tasks: The nose received, on average, slightly more fixations than did the eye regions. Also, the number of fixations significantly increased for the nose and mouth areas, but not for the eyes, when the difficulty of the task was increased; thus, the eyes were not considered as informative as in the other tasks. Additionally, the left face, or, more precisely, the nose of the left face, was looked at significantly more often than was the right face (or the nose on the right face); the first fixation in each trial was also by far more often directed to the (left) nose in the feminine task than in the other two tasks (see Figure 7A). These findings suggest that although observers referred to the sex of the face stimuli in both sex-related tasks (sex and the feminine tasks), they distributed their attention (i.e., their eye movements) differently over the face stimuli in both tasks. Hence, the question arises 
whether these differences in viewing behavior can account for the huge discrepancy in performance between the sex and the feminine tasks. This issue will be addressed in the following paragraphs.

Different viewing strategies? The strategy that participants pursued in the sex task of the present experiment seems to be suboptimal if one considers the performance for all tasks, especially the high accuracy in the feminine task. It would be interesting (but it is, however, beyond the scope of this study) to test directly whether participants can be instructed to use a specific strategy, and how that would affect their behavior. We will now discuss in more detail what the differences in strategy might relate to, in view of both the behavioral differences we found in our analyses and the other findings from related studies.

Our data show that the nose was the most scanned feature in the feminine task. The nose could indeed be diagnostic for the characterization of the sex of faces, as has been proposed by some authors (Chronicle, Chan, \& Hawkings, 1995; Roberts \& Bruce, 1988; but for different opinions, see, e.g., Pearson et al., 2003; Schyns et al., 2002). Yet, in our view, it is unlikely that observers used different features in two sex-related tasks performed during the same experiment. Moreover, as Chronicle and colleagues reported in their 1995 article, in full-frontal views, the distinctive shape of a nose is not completely available to the perceiver, making sex judgments in that orientation harder rather than easier. We would thus not expect that looking particularly at the nose should lead to notably higher performance in a sex-related task asking for the more feminine of two frontal-view images of faces.

As we already mentioned, different gaze behavior could indicate that observers directed their attention according to different spatial scales (Goffaux et al., 2005; Schyns \& Oliva, 1999). Schyns and Oliva showed flexible use of scale information for different face tasks; among other findings, they reported that both coarse and fine scales allow for "gender decisions." Since the nose coincides with the center of the face, we propose that participants showed a stronger preference for this region than for the eyes in the feminine task because they compared the faces in a more holistic (or "configural") fashion (i.e., using low-spatial-frequency information in the visual periphery) rather than by investigating single features (at high spatial frequency); hence, they fixated the center of the stimuli more often to get a "global" view of the faces. The assumption of a holistic approach is substantiated by the fact that in the postexperimental interviews, most participants reported that they had performed the feminine task in a more "intuitive" way than they had the other two tasks, and that they had tried to get an "overall impression" of one face before comparing it with the other. This orally reported strategy is further corroborated by the considerably higher number of first fixations in each trial to the nose region in the feminine task (and to the region between the two faces) and could reflect the processing of more low-spatialfrequency information than high-level local information in the other tasks - an interpretation that is reminiscent of the findings of Schyns and Oliva as well as of Goffaux and colleagues.

Results of previous studies remain uninformative about the question of how eye movements differ between holistic and feature-based perception (see, e.g., Farah et al., 1998; Tanaka \& Sengco, 1997). We suggest that the viewing behavior observed in the feminine task - that is, participants fixating in the middle of one face, presumably to get an overall configural "impression" at low resolution, and then switching to the other face to do the same-reflects a holistic viewing strategy. We are not the first to assume that the difference between a same-different and a 2AFC task using the same stimuli is a more holistic processing of the face stimuli in the latter: Beale and Keil (1995) developed their "better-likeness" task (i.e., participants must judge which of two images is the better likeness of a particular person) specifically to bias observers, in contrast with discrimination tasks, to more holistic processing. Furthermore, in a study of face perception in children and adults, Schwarzer, Huber, and Dümmler (2005) found that participants' RTs were significantly faster when a holistic strategy was used, rather than a feature-based strategy for the same task, indicating that the processing of facial features is time consuming. Faster RTs were also found in the feminine task of our present experiment than were found in the sex task using the same face stimuli. In sum, we suggest that in the case of the feminine task, participants' faster performances, their different patterns of fixation, and their oral reports about intuitive decision all indicate a holistic (i.e., low-frequency-based) rather than a featurebased (high-frequency-based) strategy.

What remains is the question about the large performance difference between both sex-related tasks. Can they be explained, as we have suggested, by more holistic versus more feature-based strategies? In the recent face-perception literature, several studies have shown that the superior performance for "own-race," as compared with "other-race," faces that is commonly found in faceidentification tasks correlates with a more holistic perception of faces of one's own race, and with a more featurebased processing of other-race faces (Michel, Caldara, \& Rossion, 2006; Michel, Corneille, \& Rossion, 2007; Michel, Rossion, et al., 2006). Thus, in the case of the other-race effect, holistic processing seems to be at least one factor that accounts for a difference in performance between the two tasks. We would propose a similar explanation for our results.

Furthermore, on the one hand, the discrimination of faces on the basis of manipulations of their sex (a characteristic that is not expected to change over a person's lifetime) might be an extremely unnatural and, therefore, difficult task. The features intuitively considered as diagnostic for sex categorization that have been reported in single-face studies might not provide information adequate for the task. On the other hand, the internal representation of a face's perceived "femaleness" might be more intuitively accessible and thus lead to more useful (possibly more low-frequency, as was proposed earlier) information processing, indicated by the different scan- 
ning patterns in our two tasks. We propose that these differences in face scanning might underlie the seeming contradiction, in the face-perception literature, between the ease with which a face is categorized as male or female, and the difficulty of a sex-discrimination task using sexmorphed stimuli. Since it does not happen very often that people whom we know change their sex, it is not surprising that discriminating faces by their sexual information is not what our visual system is tuned for.

Half-face comparison? Unexpectedly, large asymmetries were revealed in the fixation frequencies to the left and right bilateral facial features, such as the eyes and the cheeks (Figure 9). We expected observers rather to compare the left half-faces with each other, as opposed to the right half-faces, on account of the repeatedly reported lefthemiface bias in face-perception tasks (see, e.g., Butler et al., 2005; Gilbert \& Bakan, 1973; Mertens et al., 1993). Astonishingly, our results show that participants directed more fixations to the inner half of each face (nearer to the fixation point in the middle of the screen) than to the outer half, irrespective of the position of the face on the screen (left or right). This asymmetry was found in all tasks. This finding is surprising because it indicates that participants did not compare the corresponding features of the two faces - for example, the left eye of the left face with the left eye of the right face. Our scanpath analyses (see Figure 10) support this result, showing that observers made "asymmetric" comparisons of the eye regions much more frequently than they made "symmetric" comparisons (Figure 10). One could assume that this behavior is an effect of the restricted time available to respond, but the results of the pilot experiment in which participants were given an unlimited time to respond revealed exactly the same pattern (results are not shown). Reduced fixation to eccentric AOIs could be an explanation as well, but the results of other studies cast doubt on such an eccentricity effect. For example, Galpin and Underwood (2005), in a comparative visual search study, had their observers make $16.5^{\circ}$ eye movements without the possibility to move their heads, and apparently without any difficulty on their part. In the present experiment, the visual angle between the left eyes of both faces was typically about $14^{\circ}$. Furthermore, Havard and Burton (2006) found a similar tendency to focus on the inner half of two faces in a comparison task, although their faces were much smaller (about $9^{\circ} \times 6^{\circ}$ of visual angle) and separated by only $2^{\circ}$. Since Henderson and colleagues (2003) showed the perceptual span to be only $4^{\circ}$ around the fixation point at maximum, we can assume that our observers could not "see" the whole facial (particularly featural) information when fixating only on the inner half. We nevertheless tested the possibility that preferred fixation on the inner facial half might be a consequence of our setup by having another group of participants perform Experiment 1 while resting their chins on a soft foam ball to control only vertical head position; their head was not fixed, and they were explicitly instructed not to care about moving their head a little. This was possible because the iViewX eye camera can compensate for slight head movements. However, the results (not shown in the present article) remained exactly the same: Participants fixated predominantly on the inner half of the two faces they were comparing.

The simplest explanation is that humans consider faces to be generally symmetrical, even though they actually know that this is true only to a limited extent. It intuitively appears to be a useful heuristic, and the visual system seems to keep relying on it even when task difficulty increases: Our results show that when similarity between faces increased, our observers did not pay more attention to the outer halves of the face stimuli.

Observers' sex. We found surprising differences between male and female viewing strategies in both sexrelated tasks, but not in the identity-related task. In the sex and feminine tasks, female participants looked significantly more often at the eyes, whereas male participants looked more often at the cheeks of the face stimuli (independently of observers' presumably more "holistic" strategy in the feminine task, as was discussed previously). These sex-related differences in gaze behavior did not have any impact on performance, raising the question of why male and female observers looked at the faces in a different way at all.

One could speculate that in their inner representations of male and female faces, female observers place the main emphasis on the eyes (presumably including the brow region) as a characteristic feature disclosing important physiognomic differences between the sexes, whereas male observers emphasize different shape occurrences of the cheeks more (and/or maybe the outer face shape at this location). These findings are in agreement with those of previous studies that have proposed that these two face structures code for differences in male and female faces or are used by observers for sex judgments on faces (see, e.g., O'Toole et al., 1998; Yamaguchi, Hirukawa, \& Kanazawa, 1995).

A more technical aspect of these findings is that they point to the shortcoming of considering performance alone as a measure with which to investigate face processing. In our case, by relying on performance alone, we would have failed to notice sex-related differences in face processing that were revealed only in behavioral data. Obviously, it is of fundamental importance to balance observers' sex in face-perception tasks (especially when sex as a facial characteristic is involved) and to analyze the data of male and female participants separately. This is even more indicated in view of the growing evidence in the recent literature for sex-related differences in performance (see, e.g., Guillem \& Mograss, 2005; Lewin \& Herlitz, 2002; O’Toole et al., 1996; Rehnman \& Herlitz, 2007), behavior (e.g., the results of this study), and the processing of face stimuli (in terms of brain activity; e.g., Guntekin \& Basar, 2007; Kranz \& Ishai, 2006).

\section{Conclusion}

The present study demonstrates the great influence of task instruction on viewing behavior and on performance when people compare faces. Using an eyetracking technique, we could follow the observer's focus of attention 
and thus reveal which different parts of a face were considered informative with regard to the task participants had to perform. While in general confirming what has formerly been reported regarding the importance of specific facial features in single-face perception tasks, our design allows us to extend these results to the more general level of identity and sex representations of faces, independent from task-related memory references. Interestingly, our data reveal that male observers look more often at the cheeks and that female observers scan the eyes more when the sex of the faces is a relevant feature in the task they are performing. To our knowledge, such sex differences in eye movements have not been reported before. Also, eye movements as well as performance differed for sex-related tasks that primarily differed in terms of instructions given to the participants. The interpretation of those findings is that observers directed their attention according to different spatial scales in both tasks. Furthermore, surprisingly large asymmetries were found in viewing behavior due to the observers' focus of attention on the inner halves of both face stimuli, suggesting that observers regarded the faces as symmetric or, at least, that they found this approach a useful heuristic. This finding is in contrast with the general left-hemifield bias reported for faces.

\section{AUTHOR NOTE}

The present study was supported by the Max Planck Society. We thank Johannes Schultz for his support in implementing the analysis, as well as Quoc Vuong and three anonymous reviewers for helpful suggestions on the manuscript. Address correspondence to R. Armann, Max Planck Institute for Biological Cybernetics, Spemannstr. 38, 72076 Tübingen, Germany (e-mail: regine.armann@tuebingen.mpg.de).

\section{REFERENCES}

Angeli, A., Davidoff, J., \& Valentine, T. (2001). Distinctiveness induces categorical perception of unfamiliar faces. Perception, $\mathbf{3 0}$ (Suppl.), 58.

Barton, J. J. S., Radcliffe, N., Cherkasova, M. V., Edelman, J., \& INTRILIGATOR, J. M. (2006). Information processing during face recognition: The effects of familiarity, inversion, and morphing on scanning fixations. Perception, 35, 1089-1105.

BAXTER, J. C. (1970). Interpersonal spacing in natural settings. Sociometry, 33, 444-456.

Beale, J. M., \& KeIL, F. C. (1995). Categorical effects in the perception of faces. Cognition, 57, 217-239.

Blanz, V. (2000). Automatische Rekonstruktion der dreidimensionalen Form von Gesichtern aus einem Einzelbild. Dissertation zur Erlangung des Grades eines Doktors der Naturwissenschaften, EberhardKarls-Universität, Tübingen, Germany.

Blanz, V., \& VetTer, T. (1999). A morphable model for the synthesis of 3D Faces. In Proceedings of SIGGRAPH 99 (pp. 187-194). New York: ACM Press.

BRUCE, V., \& Young, A. (1986). Understanding face recognition. British Journal of Psychology, 77, 305-327.

Bruce, V., \& Young, A. (1998). In the eye of the beholder: The science of face perception. New York: Oxford University Press.

Bruyer, R., Galvez, C., \& Prairial, C. (1993). Effect of disorientation on visual analysis, familiarity decision and semantic decision on faces. British Journal of Psychology, 84, 433-441.

BüLthoff, I., \& Newell, F. N. (2004). Categorical perception of sex occurs in familiar but not unfamiliar faces. Visual Cognition, 11, 823-855

Buswell, G. T. (1935). How people look at pictures. Chicago: University of Chicago Press.

Butler, S., Gilchrist, I. D., Burt, D. M., Perret, D. I., Jones, E. \& Harvey, M. (2005). Are the perceptual biases found in chimeric face processing reflected in eye-movement patterns? Neuropsychologia, 43, 52-59.

Christman, S. D., \& Hackworth, M. D. (1993). Equivalent perceptual asymmetries for free viewing of positive and negative emotional expressions in chimeric faces. Neuropsychologia, 31, 621-624.

Chronicle, E. P., Chan, M. Y., \& Hawkings, C. (1995). You can tell by the nose: Judging sex from an isolated facial feature. Perception, 24, 969-973.

Deffenbacher, K. A., Hendrickson, C., O’Toole, A. J., Huff, D. P., \& ABDI, H. (1998). Manipulating face gender: Effects on categorization and recognition judgements. Journal of Biological Systems, $\mathbf{6}$, 219-239.

Farah, M. J., Willson, K. D., Drain, M., \& Tanaka, J. N. (1998). What is "special" about face perception? Psychological Review, 105, 482-498.

GalPin, A. J., \& UNDERWOOD, G. (2005). Eye movements during search and detection in comparative visual search. Perception \& Psychophysics, 67, 1313-1331.

Ganel, T., \& Goshen-Gottstein, Y. (2002). Perceptual integrality of sex and identity of faces: Further evidence for the single-route hypothesis. Journal of Experimental Psychology: Human Perception \& Performance, 28, 854-886.

Gilbert, C., \& BAKan, P. (1973). Visual asymmetry in perception of faces. Neuropsychologia, 11, 355-362.

GiLleland, M. (n.d.). Levenshtein distance, in three flavors. Retrieved January 2006 from www.merriampark.com/ld.htm.

Gitelman, D. R. (2002). ILAB: A program for postexperimental eye movement analysis. Behavior Research Methods, Instruments, \& Computers, 34, 605-612.

Goffaux, V., Hault, B., Michel, C., Vuong, Q. C., \& Rossion, B. (2005). The respective role of low and high spatial frequencies in supporting configural and featural processing of faces. Perception, 34, $77-86$.

Goldberg, J. H., \& Kotval, X. P. (1999). Computer interface evaluation using eye movements: Methods and constructs. International Journal of Industrial Ergonomics, 24, 631-645.

Gosselin, F., \& Schyns, P. G. (2001). Bubbles: A technique to reveal the use of information in recognition tasks. Vision Research, 41, 22612271.

Guillem, F., \& Mograss, M. (2005). Gender differences in memory processing: Evidence from event-related potentials to faces. Brain \& Cognition, 57, 84-92.

GUNTEKIN, B., \& BASAR, E. (2007). Gender differences influence brain's beta oscillatory responses in recognition of facial expressions. Neuroscience Letters, 424, 94-99.

Havard, C., \& Burton, A. M. (2006). The eye movement strategies performed during a face matching task. Perception, 35, 210.

Henderson, J. M., Williams, C. C., Castelhano, M. S., \& Falk, R. J. (2003). Eye movements and picture processing during recognition. Perception \& Psychophysics, 65, 725-734.

Henderson, J. M., Williams, C. C., \& FalK, R. J. (2005). Eye movements are functional during face learning. Memory \& Cognition, 33, 98-106.

Kanwisher, N., McDermott, J., \& Chun, M. M. (1997). The fusiform face area: A module in human extrastriate cortex specialized for face perception. Journal of Neuroscience, 17, 4302-4311.

KranZ, F., \& IshaI, A. (2006). Face perception is modulated by sexual preference. Current Biology, 16, 63-68.

LeWIN, C., \& Herlitz, A. (2002). Sex differences in face recognitionWomen's faces make the difference. Brain \& Cognition, 50, 121-128.

Loftus, G. R., \& MacKworth, N. H. (1978). Cognitive determinants of fixation location during picture viewing. Journal of Experimental Psychology: Human Perception \& Performance, 4, 565-572.

Macmillan, N. A., \& Creelman, C. D. (1991). Detection theory: A user's guide. New York: Cambridge University Press.

Mertens, I., Siegmund, H., \& Grüsser, O. J. (1993). Gaze motor asymmetries in the perception of faces during a memory task. Neuropsychologia, 31, 989-998.

Michel, C., Caldara, R., \& Rossion, B. (2006). Same-race faces are perceived more holistically than other-race faces. Visual Cognition, 14, 55-73.

Michel, C., Corneille, O., \& Rossion, B. (2007). Race categorization modulates holistic face encoding. Cognitive Science, 31, 911-924.

Michel, C., Rossion, B., Han, J., Chung, C.-S., \& Caldara, R. 
(2006). Holistic processing is finely tuned for faces of one's own race. Psychological Science, 17, 608-615.

O’Toole, A. J., Deffennacher, K. A., Valentin, D., McKee, K., HufF, D., \& ABDI, H. (1998). The perception of face gender: The role of stimulus structure in recognition and classification. Memory \& Cognition, 26, 146-160.

O’Toole, A. J., Peterson, J., \& Deffenbacher, K. A. (1996). An “otherrace effect" for categorizing faces by sex. Perception, 25, 669-676.

Pearson, A. M., Henderson, J. M., Schyns, P. G., \& Gosselin, F. (2003). Task-dependent eye movements during face perception. $A b$ stracts of the Psychonomic Society, $\mathbf{8}, 84$.

RehnMAN, J., \& Herlitz, A. (2007). Women remember more faces than men do. Acta Psychologica, 124, 344-355.

Roberts, T., \& Bruce, V. (1988). Feature saliency in judging the sex and familiarity of faces. Perception, 17, 475-481.

Rossion, B. (2002). Is sex categorization from faces really parallel to face recognition? Visual Cognition, 9, 1003-1020.

Schwarzer, G., Huber, S., \& Dümmler, T. (2005). Gaze behavior in analytical and holistic face processing. Memory \& Cognition, 33, 344-354.

Schyns, P. G., Bonnar, L., \& Gosselin, F. (2002). Show me the features! Understanding recognition from the use of visual information. Psychological Science, 13, 402-409.
Schyns, P. G., \& Oliva, A. (1999). Dr. Angry and Mr. Smile: When categorization flexibly modifies the perception of faces in rapid visual presentations. Cognition, 69, 243-265.

Stacey, P. C., Walker, S., \& Underwood, J. D. M. (2005). Face processing and familiarity: Evidence from eye-movement data. British Journal of Psychology, 96, 1-17.

TANAKa, J. W., \& SengCO, J. A. (1997). Features and their configuration in face recognition. Memory \& Cognition, 25, 583-592.

Valentine, T. (1991). A unified account of the effects of distinctiveness, inversion, and race in face recognition. Quarterly Journal of Experimental Psychology, 43A, 161-204.

Webster, M. A., Kaping, D., Mizokami, Y., \& Duhamel, P. (2004). Adaptation to natural facial categories. Nature, 428, 557-561.

Williams, L. M., Senior, C., David, A. S., Loughland, C. M., \& GoRDON, E. (2001). In search of the "Duchenne smile": Evidence from eye movements. Journal of Psychophysiology, 15, 122-127.

Yamaguchi, M. K., Hirukawa, T., \& Kanazawa, S. (1995). Judgment of gender through facial parts. Perception, 24, 563-575.

Yarbus, A. L. (1967). Eye movements and vision. New York: Plenum.

(Manuscript received April 15, 2007;

revision accepted for publication January 10, 2009.) 